\title{
BRCAness, SLFN11, and RB1 loss predict response to topoisomerase I inhibitors in triple-negative breast cancers
}

\author{
Florence Coussy ${ }^{1,2,3}$, Rania El-Botty ${ }^{1}$, Sophie Château-Joubert ${ }^{4}$, Ahmed Dahmani ${ }^{1}$, \\ Elodie Montaudon ${ }^{1}$, Sophie Leboucher ${ }^{5}$, Ludivine Morisset ${ }^{1}$, Pierre Painsec ${ }^{1}$, Laura Sourd ${ }^{1}$, \\ Léa Huguet ${ }^{1}$, Fariba Nemati ${ }^{1}$, Jean-Luc Servely ${ }^{4,6}$, Thibaut Larcher ${ }^{7}$, Sophie Vacher ${ }^{3}$, \\ Adrien Briaux ${ }^{3}$, Cécile Reyes ${ }^{1}$, Philippe La Rosa ${ }^{8,9}$, Georges Lucotte ${ }^{8,9}$, Tatiana Popova ${ }^{9,10}$, \\ Pierre Foidart ${ }^{11}$, Nor Eddine Sounni ${ }^{11}$, Agnès Noel ${ }^{11}$, Didier Decaudin ${ }^{1,2}$, Laetitia Fuhrmann ${ }^{12}$, \\ Anne Salomon ${ }^{12}$, Fabien Reyal ${ }^{13,14}$, Christopher Mueller ${ }^{15}$, Petra Ter Brugge ${ }^{16}$, Jos Jonkers ${ }^{16}$, \\ Marie-France Poupon ${ }^{1}$, Marc-Henri Stern ${ }^{9,10}$, Ivan Bièche ${ }^{3}$, Yves Pommier ${ }^{17 *}$, Elisabetta Marangoni ${ }^{1 *}$
}

Topoisomerase I (TOP1) inhibitors trap TOP1 cleavage complexes resulting in DNA double-strand breaks (DSBs) during replication, which are repaired by homologous recombination (HR). Triple-negative breast cancer (TNBC) could be eligible for TOP1 inhibitors given the considerable proportion of tumors with a defect in HR-mediated repair (BRCAness). The TOP1 inhibitor irinotecan was tested in 40 patient-derived xenografts (PDXs) of TNBC. BRCAness was determined with a single-nucleotide polymorphism (SNP) assay, and expression of Schlafen family member 11 (SLFN11) and retinoblastoma transcriptional corepressor 1 (RB1) was evaluated by real-time polymerase chain reaction (RT-PCR) and immunohistochemistry analyses. In addition, the combination of irinotecan and the ataxia telangiectasia and Rad3-related protein (ATR) inhibitor VE-822 was tested in SLFN11-negative PDXs, and two clinical non-camptothecin TOP1 inhibitors (LMP400 and LMP776) were tested. Thirty-eight percent of the TNBC models responded to irinotecan. BRCAness combined with high SLFN11 expression and RB1 loss identified highly sensitive tumors, consistent with the notion that deficiencies in cell cycle checkpoints and DNA repair result in high sensitivity to TOP1 inhibitors. Treatment by the ATR inhibitor VE-822 increased sensitivity to irinotecan in SLFN1 1-negative PDXs and abolished irinotecan-induced phosphorylation of checkpoint kinase 1 (CHK1). LMP400 (indotecan) and LMP776 (indimitecan) showed high antitumor activity in BRCA1-mutated or BRCAness-positive PDXs. Last, low SLFN1 1 expression was associated with poor survival in 250 patients with TNBC treated with anthracycline-based chemotherapy. In conclusion, a substantial proportion of TNBC respond to irinotecan. BRCAness, high SLFN11 expression, and RB1 loss are highly predictive of response to irinotecan and the clinical indenoisoquinoline TOP1 inhibitors.

\section{INTRODUCTION}

Derivatives of the natural alkaloid camptothecin have been used in cancer therapy for more than 20 years (1). Topotecan and irinotecan are the two clinically approved topoisomerase I (TOP1) inhibitors, and irinotecan has been widely used against several cancers $(1,2)$. Camptothecin derivatives bind to the DNA-TOP1 cleavage complex

\footnotetext{
${ }^{1}$ Translational Research Department, Institut Curie, PSL Research University, 75005 Paris, France. ${ }^{2}$ Medical Oncology Department, Institut Curie, PSL Research University, 75005 Paris, France. ${ }^{3}$ Genetics Department, Institut Curie, PSL Research University, 75005 Paris, France. ${ }^{4}$ BioPôle Alfort, Ecole Nationale Vétérinaire d'Alfort, 94704 Maisons Alfort, France. ${ }^{5}$ Institut Curie, PSL Research University, UMR3306, 91405 Orsay, France. ${ }^{6}$ INRA, PHASE Department, 37380 Nouzilly, France. ${ }^{7}$ INRA, APEX-PAnTher, Oniris, 44300 Nantes, France. ${ }^{8}$ INSERM, U900, 75005 Paris, France. ${ }^{9}$ Institut Curie, PSL Research University, 75005 Paris, France. ${ }^{10}$ INSERM U830, 75005 Paris, France. ${ }^{11}$ Laboratory of Tumor and Developmental Biology, Groupe Interdisciplinaire de Génoprotéomique Appliqué-Cancer (GIGA-Cancer), University of Liège, Liège 4000 , Belgium. ${ }^{12} \mathrm{De}-$ partment of Pathology, Institut Curie, PSL Research University, 75005 Paris, France. ${ }^{13}$ Surgery Department, Institut Curie, PSL Research University, 75005 Paris, France. ${ }^{14}$ U932, Immunity and Cancer, INSERM, Institut Curie, 75005 Paris, France. ${ }^{15}$ Queen's Cancer Research Institute, Queen's University, Kingston, ON K7L 3N6, Canada. ${ }^{16}$ Division of Molecular Pathology and Cancer Genomics Centre Netherlands, Netherlands Cancer Institute, Amsterdam, $1066 \mathrm{CX}$, Netherlands. ${ }^{17}$ Developmental Therapeutics Branch and Laboratory of Molecular Pharmacology, Center for Cancer Research, National Cancer Institute, National Institutes of Health, Bethesda, MD 20892, USA. *Corresponding author. Email: elisabetta.marangoni@curie.fr (E.M.); pommier@ nih.gov (Y.P.)
}

(TOP1cc) preventing religation of DNA and resulting in DNA doublestrand breaks (DSBs) during replication. These breaks cause cell cycle arrest in $S$ and $G_{2}$ phases to allow DNA repair and are followed by cell death in the absence of DNA repair. Similar to TOP2 inhibitors (anthracyclines) and poly(adenosine $5^{\prime}$-diphosphate-ribose) polymerase (PARP) inhibitors, TOP1 inhibitors trap their target enzyme on DNA and prevent the release of TOP1ccs, leading to stalled replication forks and DNA DSBs $(2,3)$.

Responses to DNA damage induced by TOP1 inhibitors are coordinated by the ataxia telangiectasia and Rad3-related protein (ATR)-CHK1 cell cycle checkpoint, which is activated by replicationinduced DSBs and replication stress $(4,5)$. At the chromatin level, $\mathrm{H} 2 \mathrm{AX}$ is phosphorylated and generates foci around DSBs. Recently, an additional replicative damage response was found, Schlafen family member 11 (SLFN11), which works independently of the ATR-CHK1 cascade, preventing the cells from progressing through the cell cycle when they accumulate DNA damage and replication stress (4).

At least two main pathways repair TOP1-mediated DNA damage: excision repair by tyrosyl-DNA phosphodiesterase and homologous recombination (HR) (5). Defects in DNA repair and checkpoints are associated with enhanced sensitivity to TOP1 inhibitors (6). Nevertheless, no biomarker has been validated for use in the clinical setting, although several biomarkers of response or resistance to 
TOP1 inhibitors have been suggested in preclinical and translational studies. These include genomic alterations in DNA repair genes and overexpression of SLFN11 as candidate biomarkers of response and overexpression of the ATP binding cassette subfamily G member 2 (ABCG2) transporter as a potential biomarker of resistance $(7,8)$.

Irinotecan is one of the main cytotoxic agents used for first-line treatment of metastatic colorectal cancer, and it is also given to patients with pancreatic and lung cancers. In breast cancer (BC), only a few studies have been published on the clinical activity of irinotecan. These trials, conducted in unselected patients with metastatic BC pretreated with anthracyclines and/or taxanes, have generated heterogeneous results (9).

Triple-negative $\mathrm{BC}$ (TNBC) is the most logical subtype for exploring TOP1 inhibitors given its high proportion of tumors with HR deficiency (HRD or BRCAness). However, preclinical and clinical studies investigating TOP1 inhibitors in TNBC are rare. In the present study, we hypothesized that TNBC carrying BRCA1/2 mutations or characterized by HRD would respond to irinotecan. We evaluated its antitumor activity in a large panel of TNBC patientderived xenografts (PDXs), molecularly characterized with the HRDlarge-scale state transition (HRD-LST) assay to measure BRCAness. Additional potential markers related to DNA damage checkpoints, retinoblastoma transcriptional corepressor 1 (RB1) and SLFN11, were identified through transcriptomic analysis and validated at the protein level. We also show that in the absence of SLFN11, response to irinotecan can be increased by adding an ATR inhibitor and that the clinical TOP1 inhibitors (indenoisoquinoline derivatives) (10-12) are highly efficient in BRCA1-mutant and BRCAnesspositive TNBC PDXs.

\section{RESULTS}

\section{A substantial proportion of TNBC PDXs respond to the TOP1 inhibitor irinotecan}

We evaluated the antitumor activity of the Food and Drug Administrationapproved TOP1 inhibitor irinotecan in 40 PDXs of TNBC $(13,14)$. Patients' clinical characteristics are summarized in Table 1 and data file S1.

Fifteen models (37.5\%) achieved complete or partial response (R), 9 (22.5\%) stable disease (SD), and 16 (40\%) showed progressive disease (PD) (Fig. 1). Examples of three PDXs that responded with $\mathrm{PD}, \mathrm{R}$ (complete response), and SD are shown in Fig. 1B. The response rate to irinotecan in PDXs was then analyzed with respect to clinical markers of poor survival in the corresponding patients, such as distant relapse after surgery and persistence of residual tumor after neoadjuvant chemotherapy. Among the 24 PDXs established from treatment-naïve tumors, $10(42 \%)$ were responders and 8 (33\%) showed SD. Among the 16 PDXs established from residual tumors after neoadjuvant chemotherapy, 5 (31\%) responded, 1 (6\%) scored as SD, and $10(63 \%)$ as PD (Fig. 1C). Of the 17 PDXs corresponding to patients who had relapsed after surgery, $9(53 \%)$ responded and 3 (18\%) scored as SD (Fig. 1D). Overall, these results suggest that irinotecan could be an effective therapy in more than one-third of TNBC including tumors that become metastatic or that show only partial response to neoadjuvant chemotherapy.

\section{Response to irinotecan is associated with BRCA1/2 mutations and BRCAness}

Because alterations in genes involved in DNA repair are associated with sensitivity to TOP1 inhibitors (2), we analyzed the response to
Table 1. Histopathological and clinical features of TNBC.

\begin{tabular}{|c|c|c|}
\hline Characteristic & $N$ & $\%$ \\
\hline \multicolumn{3}{|l|}{ Type of graft } \\
\hline Primary BC & 22 & $55 \%$ \\
\hline Primary nodes of BC & 2 & $5 \%$ \\
\hline Residual tumors after neoadjuvant & 16 & $40 \%$ \\
\hline Mean age at diagnosis & $56(29-89)$ & \\
\hline \multicolumn{3}{|l|}{ TNM } \\
\hline T1 & 8 & $20 \%$ \\
\hline T2 & 19 & $48 \%$ \\
\hline T3 & 9 & $23 \%$ \\
\hline T4 & 2 & $5 \%$ \\
\hline No & 23 & $58 \%$ \\
\hline N1 & 15 & $38 \%$ \\
\hline N2 & 1 & $3 \%$ \\
\hline Mo & 39 & $98 \%$ \\
\hline M1 & 1 & $3 \%$ \\
\hline \multicolumn{3}{|l|}{ Breast surgery } \\
\hline Tumorectomy & 16 & $40 \%$ \\
\hline Mastectomy & 24 & $60 \%$ \\
\hline \multicolumn{3}{|l|}{ Lymph node surgery } \\
\hline Sentinel node biopsy & 6 & $15 \%$ \\
\hline Lymphadenectomy & 33 & $83 \%$ \\
\hline \multicolumn{3}{|l|}{ Histology } \\
\hline Invasive carcinoma of no special type & 37 & $93 \%$ \\
\hline Metaplastic BC & 3 & $8 \%$ \\
\hline Lymphovascular invasion (LVI) $^{+}$ & 14 & $35 \%$ \\
\hline $\mathbf{L V I}^{-}$ & 26 & $65 \%$ \\
\hline \multicolumn{3}{|l|}{ Rank SBR } \\
\hline Grade SBR 1 & 0 & $0 \%$ \\
\hline Grade SBR 2 & 1 & $3 \%$ \\
\hline Grade SBR 3 & 39 & $98 \%$ \\
\hline \multicolumn{3}{|l|}{ Recurrence } \\
\hline No relapse & 15 & $37.5 \%$ \\
\hline Local relapse & 8 & $20 \%$ \\
\hline Distant relapse & 17 & $42.5 \%$ \\
\hline
\end{tabular}

irinotecan in relation to mutations in the $B R C A 1$ and $B R C A 2$ genes, previously characterized in the PDXs (15). Pathogenic somatic mutations in BRCA1 or BRCA2 genes were present in $28 \%$ of PDXs (11 of 40), with 6 in the R group (40\%), 4 in the SD group (44\%), and 1 in the PD group (6\%) (Fig. 2, A and B). The association between BRCA1/2 mutation and response (including both $S D$ and $\mathrm{R}$ ) was statistically significant ( $P=0.027$, Fisher's exact test) (Fig. 2B). We next analyzed the response to irinotecan as a function of the BRCAness status, determined by the LSTs genomic signature $(16,17)$. Overall, 70\% (28 of 40) of the TNBC PDXs showed BRCAness. Of these, 11 were BRCA1 or BRCA2 mutated, 10 were BRCA1 methylated, and 6 were of unknown origin. Twenty-one PDXs showed BRCAness 
A Response to irinotecan treatment in 40 TNBC PDXs

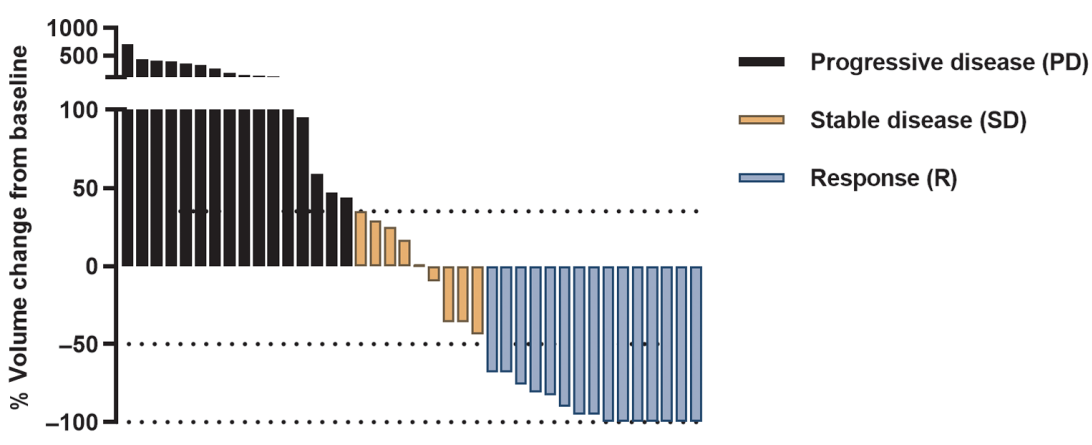

B

HBCx-39 (PD)

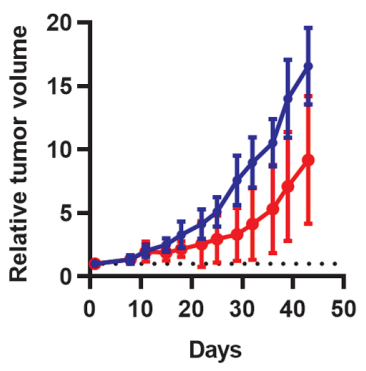

C

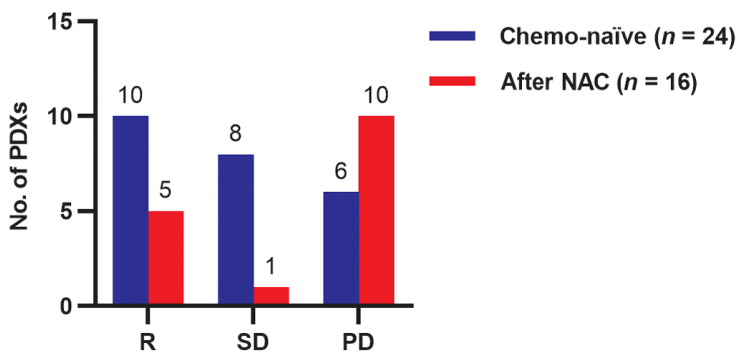

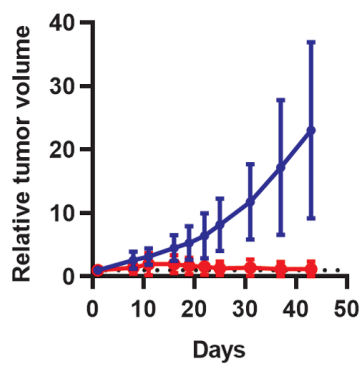

D
$\mathrm{HBC}-4 \mathrm{~B}(\mathrm{R})$
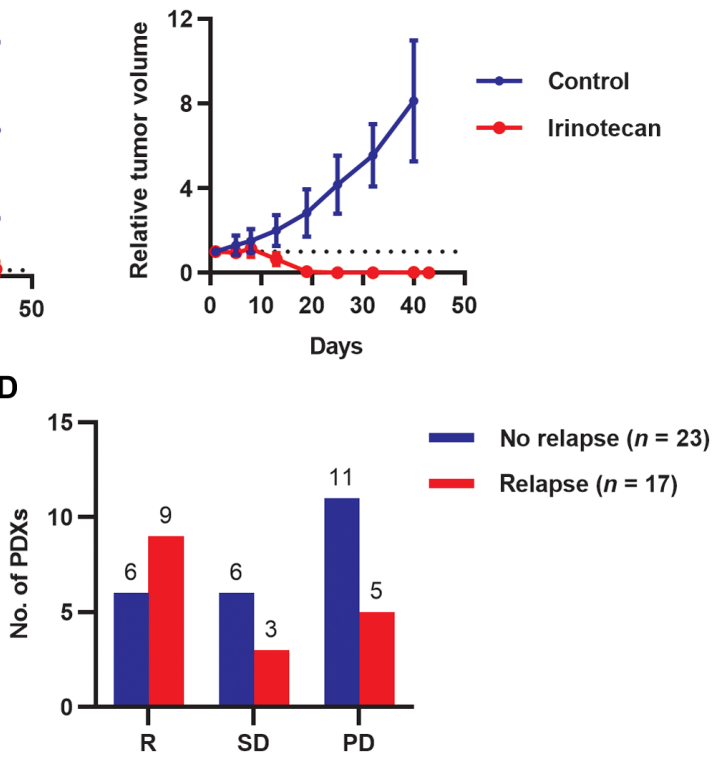

Fig. 1. Response to irinotecan in TNBC PDXs. (A) Waterfall plot representing responses to irinotecan treatment in 40 TNBC PDXs. Each bar represents the median change in tumor volume from baseline in treated xenografts; $n=4$ to 13 xenografts per group. (B) Irinotecan response in $H B C x-39, H B C x-10$, and $H B C x-4 B$. Means \pm standard deviation, $n=8$ to 13. (C) Response rates (number of PDXs) to irinotecan according to patients' pretreatment. NAC, neoadjuvant chemotherapy. (D) Response rates to irinotecan according to patients' distant relapse after surgery for the primary tumor.

in the group of $\mathrm{R}+\mathrm{SD}(88 \%)$, whereas only seven showed BRCAness in the PD group (44\%) $(P=0.005$, Fisher's exact test; Fig. 2, A and B). BRCA $1 / 2$ mutations, LST status, and BRCA1 methylation status for each PDX are provided in data file S2.

The LST signature of BRCAness is associated with a deficiency in HR-mediated DNA repair $(16,18)$. To assess DNA damage and repair after irinotecan treatment, we performed a pharmacodynamics study in two PDXs, Human Breast Cancer xenograft 60 (HBCx-60) (BRCAness, highly sensitive to irinotecan) and HBCX-106 (no BRCAness, resistant to irinotecan) (Fig. 2C). DNA damage, measured by Western blot analysis of $\gamma \mathrm{H} 2 \mathrm{AX}$, was increased in treated tumors of the irinotecan responder $\mathrm{HBCX}-60$ model but not in the irinotecanresistant HBCx-106 xenografts (Fig. 2D). RAD51 foci, in tumor cells expressing the $\mathrm{S}-\mathrm{G}_{2}$ cell cycle marker geminin, have been proposed as a functional assay of HR-mediated repair in primary BC (19).

Immunofluorescence analysis of RAD51 foci in HBCX-106 xenografts 24 hours after irinotecan treatment showed a high number of geminin-positive cells with at least 10 RAD51 foci (mean 61\%); whereas in the $\mathrm{HBCx}-60$ xenograft, there were very few gemininpositive cells with RAD51 foci (mean 1\%, Fig. 2E). A representative picture of geminin-positive cells in the two tumors is shown in Fig. 2F. These results demonstrate that response to irinotecan in TNBC PDXs is strongly correlated with BRCA1/2 mutations and with the LST signature of BRCAness.

\section{SLFN1 1 expression and RB loss are potential markers of irinotecan response}

To identify additional potential markers of irinotecan response, we analyzed the previously generated transcriptomic profiles of TNBC PDXs (14). A differential expression analysis of transcriptomic datasets comparing responder (R) and resistant PDXs (PD) identified 282 differentially expressed genes (data file S3). One of the top up-regulated genes in responder PDXs is SLFN11 (Fig. 3, A and B). Expression of SLFN11 is positively correlated with response to TOP1 inhibitors, and a lack of its expression is associated with drug resistance in the NCI-60 and the Broad Institute Cancer Cell 
Fig. 2. Response to irinotecan and BRCAness. (A) Waterfall plot representing irinotecan responses in PDXs with (green) and without BRCAness (gray). BRCA1/2 mutations and BRCA1 methylation are indicated with * and $M$, respectively. (B) Contingency analysis of BRCA $1 / 2$ mutations and BRCAness (Fisher's exact test). $\mathrm{R}$, response; $\mathrm{SD}$, stable disease; $P D$, progressive disease; $w t$, wild type. (C) Response to irinotecan in $\mathrm{HBCx}-60$ (BRCAness) and $\mathrm{HBC} x-106$ (no BRCAness). $n=4$ to 5 , means \pm standard deviation. (D) Western blot analysis of $\gamma \mathrm{H} 2 \mathrm{AX}$ in $\mathrm{HBCx}-60$ and $\mathrm{HBCx}-106$ xenografts, 4 and 24 hours after a single treatment with irinotecan $(n=3)$. (E) Percentage of geminin-positive nuclei with more than 10 RAD51 foci in HBCX-106 and HBCX-60 xenografts (control and irinotecan-treated groups, tumors harvested 24 hours after a single treatment); $n=3$. ns, not significant. (F) Representative images showing RAD51 foci (green) and geminin (red) immunofluorescence in $\mathrm{HBCX}-106$ and $\mathrm{HBCx}-60$ xenografts harvested 24 hours after a single irinotecan treatment. Scale bars, $10 \mu \mathrm{m}$. DAPI, 4',6-diamidino-2phenylindole.

Line Encyclopedia panels $(7,20)$. The $R B 1$ gene was among the down-regulated genes in the responder tumors (Fig. 3, A and B, and data file S3). TOP1 and $A B C B 1$ (MDR1), two genes potentially linked to irinotecan response, were not differentially expressed in responder versus resistant PDXs (fig. S1).

SLFN11 acts as an S-phase checkpoint, preventing cancer cells from progressing and surviving when they accumulate DNA damage and replication stress $(4,21)$. Its expression in $\mathrm{BC}$ had not been studied until recently (22). In the TNBC subtype in The Cancer Genome Atlas (TCGA), SLFN11 gene has a wide range of expression with a bimodal distribution, potentially designating a subgroup with relatively high expression of SLFN11 (fig. S2A).

We validated $S L F N 11$ and $R B 1$ expression at the gene level by real-time polymerase chain reaction (RT-PCR) in PDXs and confirmed that SLFN11 and RB1 gene expression were higher and lower, respectively, in the group of responding tumors (Fig. 3C). Next, we analyzed SLFN11 expression by immunohistochemistry (IHC). Three examples are shown in Fig. 3D. SLFN11 gene and protein

$A$

D

E

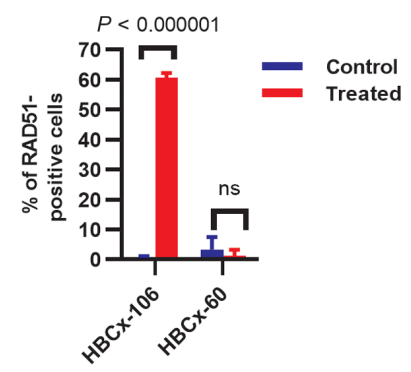

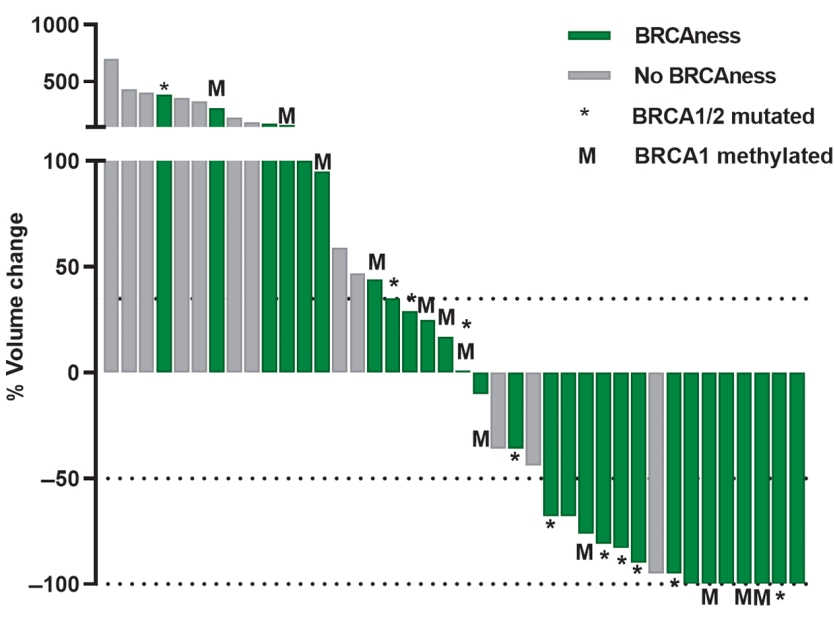
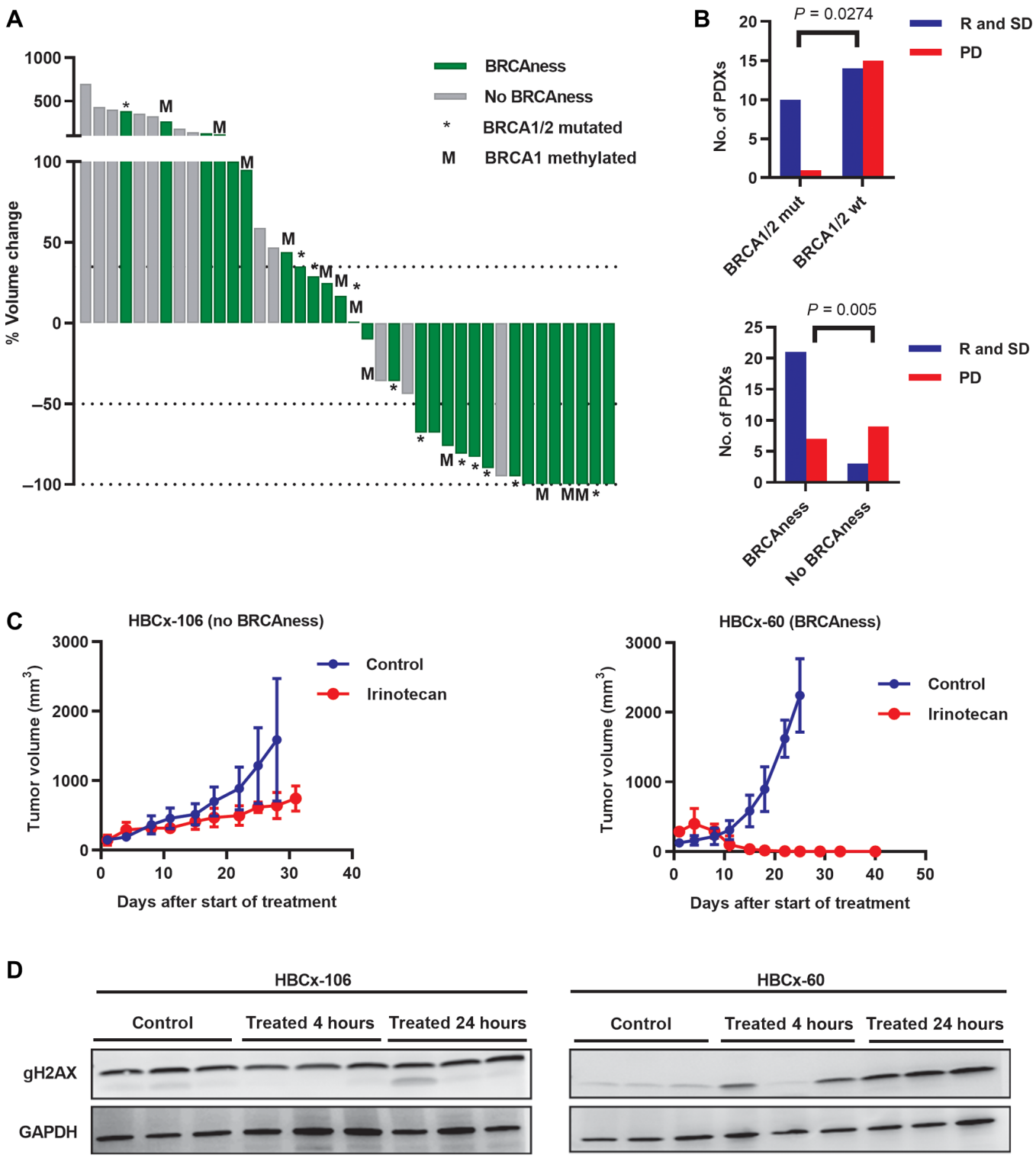

$\mathbf{F}$

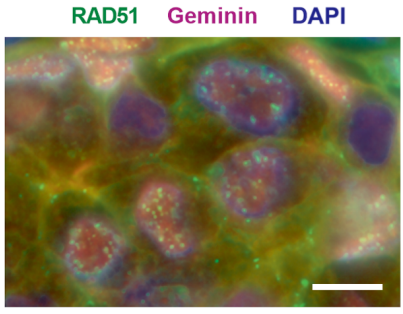

HBCx-106
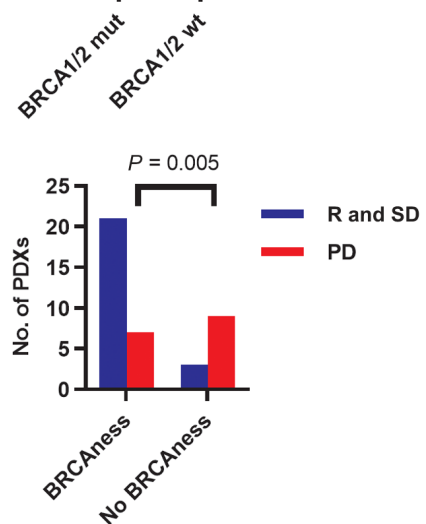

expression $(H$-score $)$ were significantly correlated $(P<0.0001$, Fig. 3E). Among the 40 PDXs, 19 were SLFN11 negative $(H$-score $=0)$, 10 had a low $H$-score (range between 4 and 40 ), and 11 had a high $H$-score (range between 75 and 285). Figure 3F shows the frequency distribution of SLFN11 expression across the PDXs. SLFN11 expression was significantly different between $\mathrm{R}$ and $\mathrm{SD}+\mathrm{PD}$ groups 

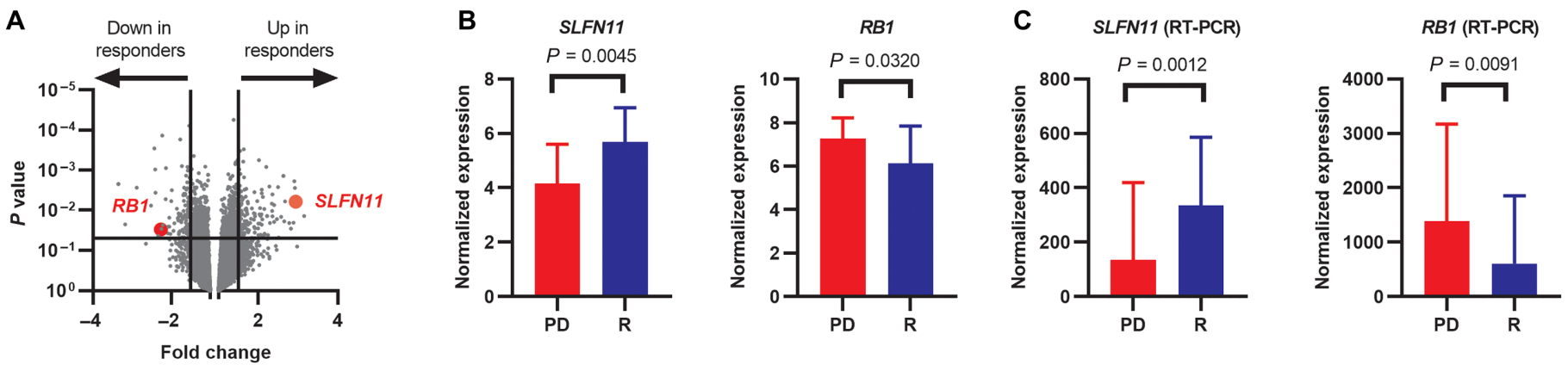

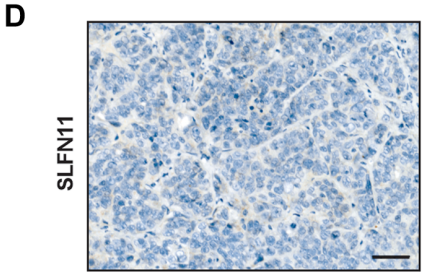

HBCx-39

F

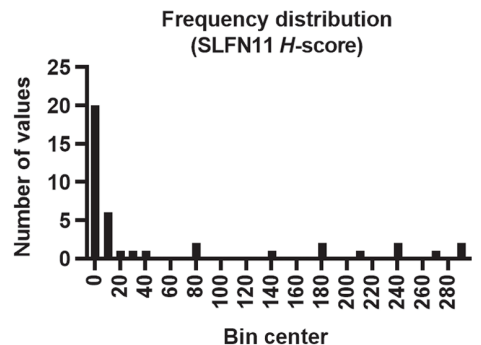

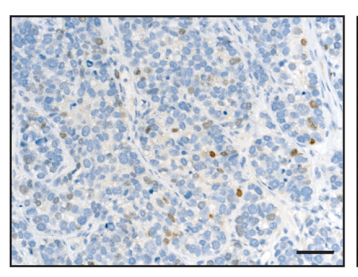

HBCx-40

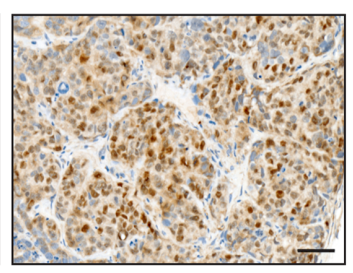

HBCx-14
E

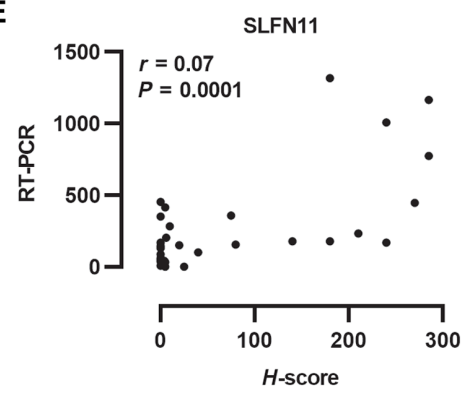

G

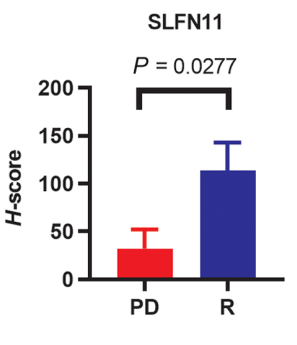

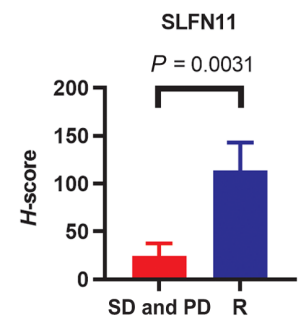

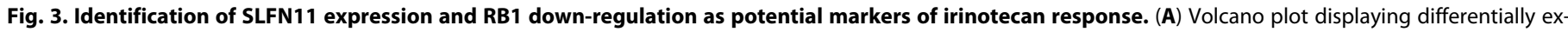

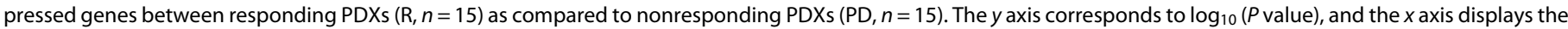

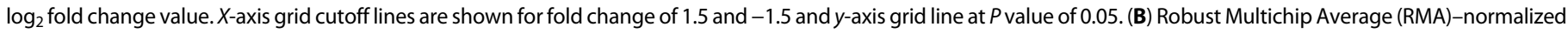

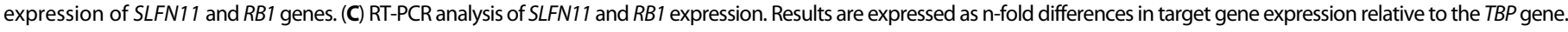

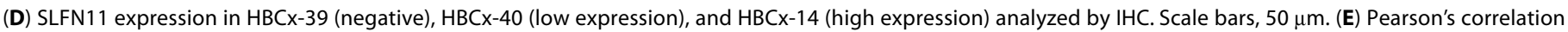

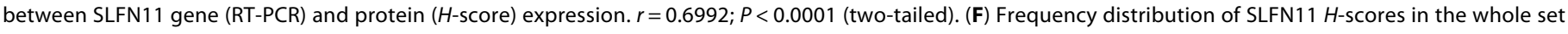
of PDXs. (G) SLFN1 $1 \mathrm{H}$-scores in PD as compared to R and in PD and SD categories as compared to R.

$(P=0.0031$; Fig. $3 \mathrm{G})$ and between $\mathrm{R}$ and $\mathrm{PD}$ groups $(P=0.0277$; Fig. 3G). Of the 15 responding PDXs, 8 (53\%) had high SLFN11 expression ( $H$-score between 80 and 285$)$, and 7 (47\%) were SLFN11 low or negative. By contrast, in the group of SD and PD (25 PDXs), only $3(12 \%)$ were SLFN11 high, and $22(88 \%)$ were SLFN11 low or negative $(P=0.009$, Fisher's exact test) (Fig. 4A). Sensitivity and specificity of high expression of SLFN11 as a potential marker were 53 and $88 \%$, respectively.

Next, we analyzed the predictive value of BRCAness combined with SLFN11 expression. Nine PDXs scored as BRCAness positive and SLFN11 high (22.5\%), and all of them were in the responder group (corresponding to $60 \%$ of responder tumors) (Fig. 4B). BRCAness combined with high SLFN11 expression identified a subgroup of tumors highly sensitive to irinotecan $(P<0.0001$; Fisher's exact $t$ test). Sensitivity and specificity of BRCAness combined with high $H$-score of SLFN11 in predicting irinotecan response were 60 and $100 \%$, respectively (Fig. 4C). To determine the fraction of patients presenting with this combination of biomarkers, we analyzed BRCAness and SLFN11 gene expression (based on $z$ score) in the TCGA cohort of TNBC and found that $22 \%$ of tumors had high SLFN11 expression and a BRCAness phenotype (fig. S2B). The
BRCAness phenotype in TCGA TNBC was due to BRCA1 and BRCA2 germline mutations (18 and $3 \%$, respectively), to $B R C A 1 / 2$ somatic mutations in $7 \%$ of tumors, to BRCA1 methylation (30\%), to BRCA1 missense mutations (5\%), or to RAD51 mutations or methylation (14\%) (fig. S2C).

The individual tumor growth curves of five TNBC PDXs with complete response in all treated animals are shown in Fig. 4D. Matching SLFN11 expression is shown in Fig. 4E. The PDXs HBCx-15 and $\mathrm{HBCX}-66$ were resistant to olaparib.

Next, we assessed the down-regulation of $R B 1$ because $R B 1$ is frequently inactivated in TNBC and is a DNA damage checkpoint whose inactivation has been linked to increased sensitivity to many chemotherapies, including TOP1 inhibitors (23-27). RB1 expression was determined by IHC analysis in TNBC PDXs in two previous works $(14,28)$. Among the 40 PDXs, 15 had RB1 loss (38\%), 10 in the $\mathrm{R}$ group, and 5 in the SD/PD group $(P=0.0062$, Fisher's exact $t$ test) (Fig. 4F). Sensitivity and specificity of RB1 loss as a potential biomarker of irinotecan response were 67 and 80\%, respectively. The combination of BRCAness and RB1 loss had a predictive value of $71 \%$ and sensitivity and specificity of 67 and $84 \%$, respectively $(P=0.002)($ Fig. 4G). 
A

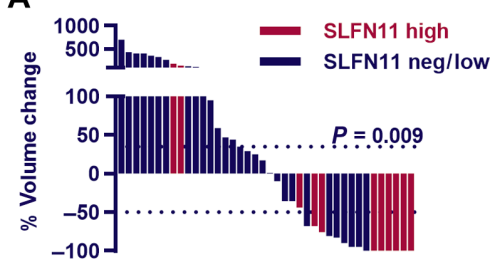

B

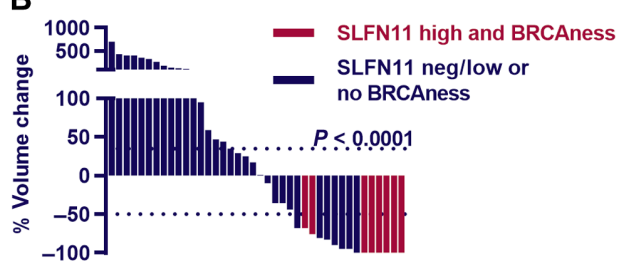

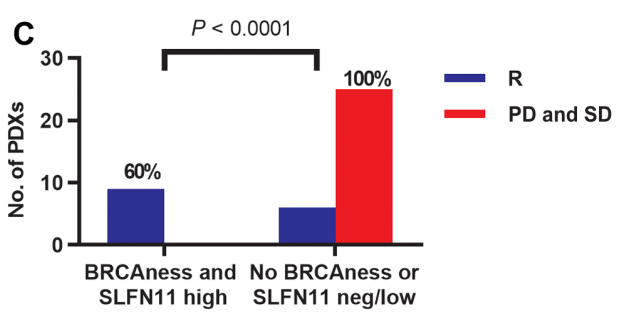

HBCx-66 (BRCA1 mutated)
D $\begin{gathered}\text { HBC-x60 } \\ \text { (BRCAness) }\end{gathered}$

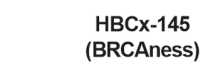

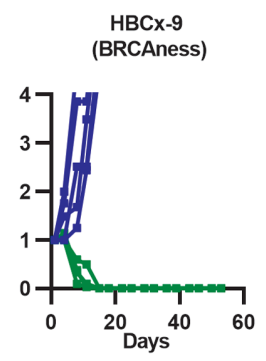
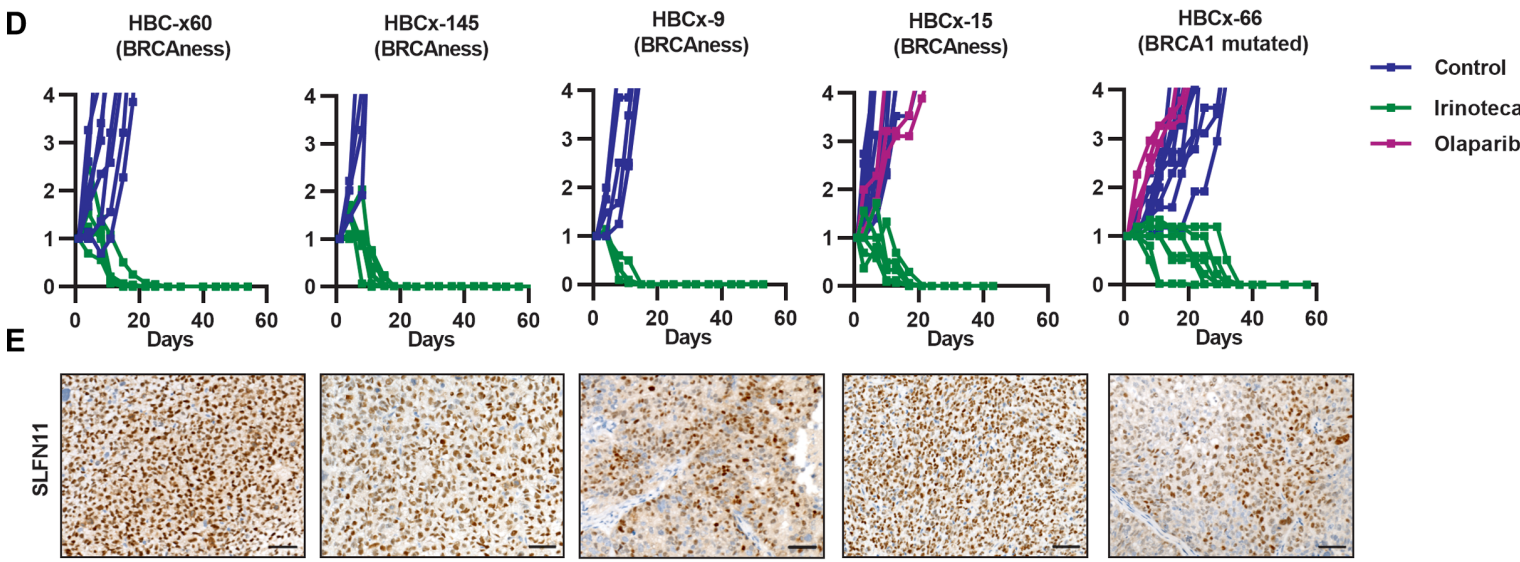

$\mathbf{F}$
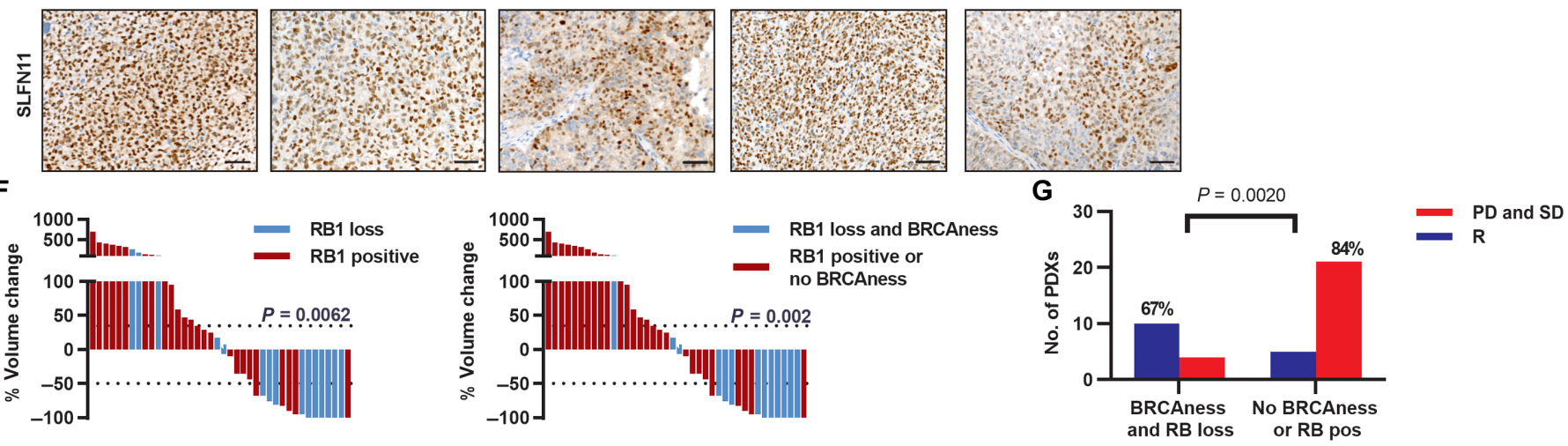

Fig. 4. Combination markers correlated with irinotecan response. (A) A waterfall plot showing SLFN11 expression and irinotecan response. (B) Combination of SLFN1 1 expression and BRCAness as potential markers of response to irinotecan. (C) Contingency analysis, Fisher's exact test. (D) Individual tumor growth curves of HBCX-60, HBCX-145, HBCX-9, HBCX-15, and HBCx-66 xenografts treated with irinotecan ( $n=5$ to 10$)$ and olaparib for HBCx-15 and HBCX-66 PDXs ( $n=3$ ). (E) SLFN11 expression in $\mathrm{HBCx}-60, \mathrm{HBCx}-145, \mathrm{HBCx}-9, \mathrm{HBCx}-15$, and HBCx-66. Scale bars, $50 \mu \mathrm{m}$. (F) Waterfall plots showing irinotecan response, RB1 loss determined by IHC, and BRCAness. (G) Contingency analysis, Fisher's exact test.

Hence, in addition to BRCAness, we identified two potential markers associated with irinotecan response in TNBC, SLFN11 expression, and RB1 loss. When combined with BRCAness, both SLFN11 high $H$-score and RB1 loss identified a subgroup of tumors highly sensitive to irinotecan.

\section{Inhibition of the S-phase checkpoint with an ATR inhibitor increases response to irinotecan in SLFN11-negative xenografts} Because ATR inhibition can reverse resistance to replicative damage in SLFN11-negative cells (4), we hypothesized that inhibition of ATR could increase sensitivity to irinotecan in SLFN11-negative xenografts. The efficacy of irinotecan combined with the ATR inhibitor VE-822 (berzosertib; VX-970) was tested in two SLFN11negative PDXs with BRCAness (Fig. 5A). Treatment with irinotecan and VE-822 significantly increased tumor growth inhibition in both PDXs as compared to irinotecan alone $(P=0.0043)$.

Cellular responses to DNA damage are regulated by the ATMCHK2 and ATR-CHK1 signaling cascades upon activation by DSBs and single-stranded DNA (replication stress), respectively (29). To determine activation and inhibition of ATM-CHK2 and ATR-CHK1 cascades in treated $\mathrm{HBCx}-1$ and $\mathrm{HBCx}-23$ xenografts, we analyzed
CHK1 and CHK2 phosphorylation (Fig. 5B). CHK1 phosphorylation, a marker of ATR-CHK1 signaling activation, which was absent in control and VE-822-treated tumors, was strongly increased in irinotecan-treated tumors and completely abolished in tumors treated with the combination of irinotecan + VE-822. In contrast, CHK2 phosphorylation was not increased by irinotecan treatment (Fig. 5B). These results show that in the absence of SLFN11, a combination of irinotecan with an ATR inhibitor increases drug sensitivity in a tumor with BRCAness and that this effect is associated with impaired activation of the S-phase checkpoint by ATR-CHK1.

\section{Efficacy of non-camptothecin TOP1 inhibitors (indenoisoquinolines) in SLFN11 PDXs}

Because of the limitations of camptothecin-derived chemotherapies, non-camptothecin TOP1 inhibitors have been developed $(10,12,30)$. Thus, we tested the antitumor activity of two clinically advanced indenoisoquinoline TOP1 inhibitors LMP400 (indotecan) and LMP776 (indimitecan) in four PDXs: HBCx-60 (BRCAness, SLFN11 high, and RB1 loss), HBCX-8 (BRCA1 mutated, SLFN11 negative, and RB1 positive), $\mathrm{HBCx}-10$ (BRCA2 mutated, SLFN11 negative, and RB1 negative), and HBCx-39 (no BRCAness, SLFN11 negative, and 
A

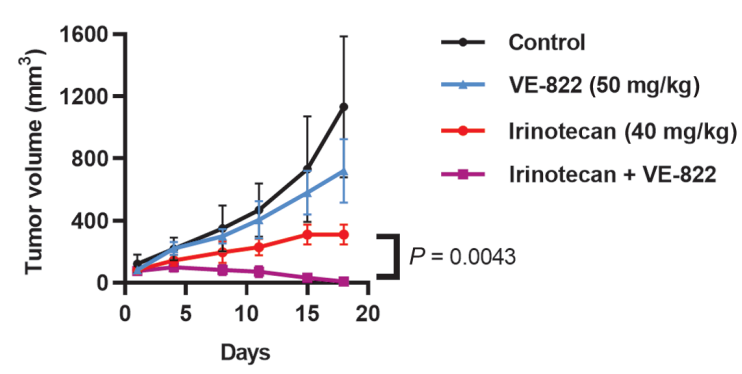

B

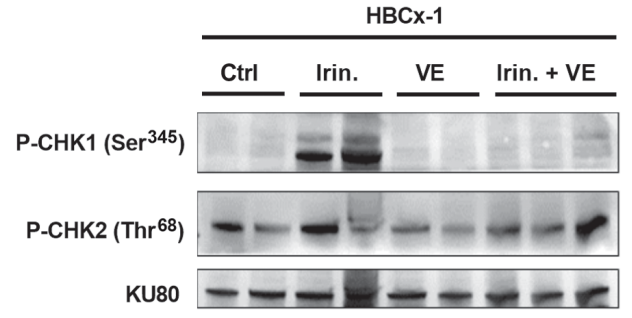

HBCx-23
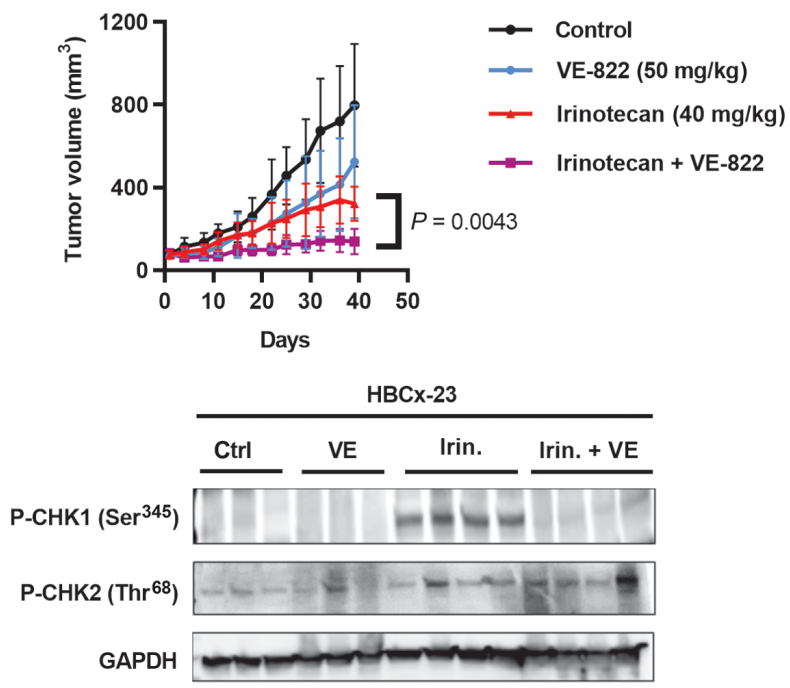

C
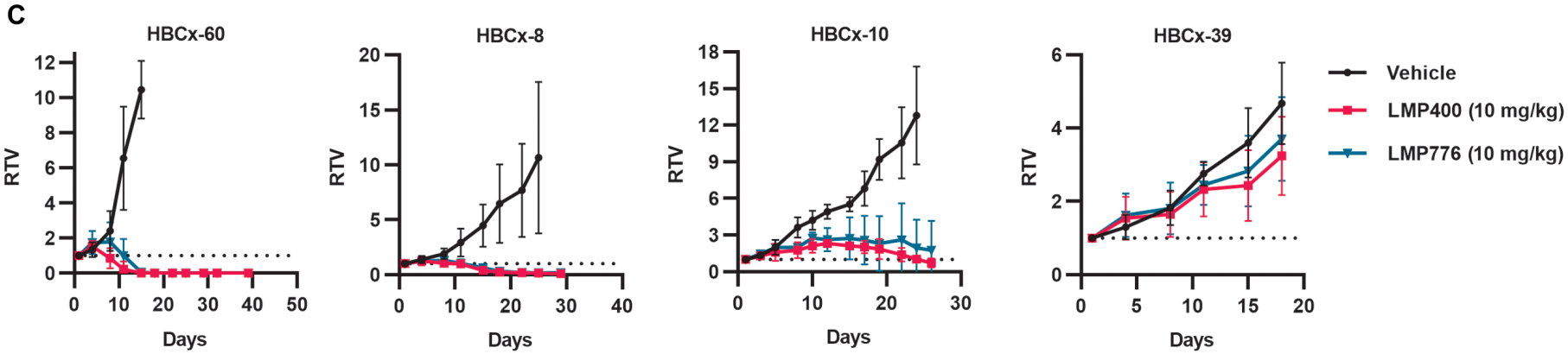

Fig. 5. Combination of irinotecan with an ATR inhibitor and antitumor activity of indenoisoquinolines. (A) Tumor response to irinotecan (40 $\mathrm{mg} / \mathrm{kg}$ ) and the $A T R$ inhibitor VE-822 (VX-870, berzosertib; $50 \mathrm{mg} / \mathrm{kg}$ ) in the HBCX-1 and HBCX-23 PDXs (BRCAness positive and SLFN11 negative); $n=4$ for control, irinotecan, and VE-822treated groups; $n=7$ for the combination group $(\mathrm{HBCX}-1)$; and $n=8$ for the $\mathrm{HBCx}-23$ xenograft groups. Statistical significance of the difference between irinotecan and irinotecan + VE-822-treated groups was determined by the Mann-Whitney test. (B) Western blot analysis of P-CHK1 (Ser ${ }^{345}$ ), P-CHK2, and KU80 or glyceraldehyde-3-phosphate dehydrogenase (GAPDH) in treated xenografts after a single dose of irinotecan (tumors harvested 24 hours after irinotecan treatment) alone or associated with two injections of VE-822 (administered at days 1 and 2, tumors harvested 4 hours after the second VE-822 treatment); $n=2$ for control, irinotecan, and VE-822 xenografts; $n=3$ for irinotecan + VE-822 xenografts (HBCX-1); and $n=3$ or 4 xenografts for $\mathrm{HBCx}-23$. (C) Antitumor activity of the indenoisoquinolines LMP400 (indotecan) and LMP776 (indimitecan) in the $\mathrm{HBCx}-60, \mathrm{HBCx}-8, \mathrm{HBCx}-10$, and $\mathrm{HBCx}-39$ PDXs. Means \pm standard deviation, $n=5$ to 6 . RTV, relative tumor volume.

RB1 positive). In the $\mathrm{HBCx}-60$ and $\mathrm{HBCx}-8$ models, both compounds showed marked antitumor activity that resulted in complete response in all treated xenografts (Fig. 5C). The HBCx-10 model responded with SD. By contrast, the two compounds did not show any antitumor activity in the HBCx-39 PDX (Fig. 5C).

\section{BRCAness, high SLFN1 1 expression, and RB1 loss predict sensitivity to anthracycline-based chemotherapy}

Because anthracycline-based chemotherapy regimens are the current standard of care for neoadjuvant and adjuvant treatments of early TNBC (31), and because the identified markers are potentially relevant for TOP2 inhibitors $(5,21)$, we compared irinotecan responses to the response to doxorubicin (Adriamycin) combined with cyclophosphamide (AC), previously determined in 39 PDXs (14). Responses to $\mathrm{AC}$ and irinotecan were similar in frequency (Fig. 6A). All the irinotecan-resistant tumors were cross-resistant to AC, and most of the irinotecan responder PDXs (13 of 14) responded to AC. Accordingly, high expression of SLFN11 and RB1 loss were also significantly associated with response to $\mathrm{AC}(P=0.019$ and $P=0.013$, respectively), and when combined with BRCAness, they predicted response with a positive predictive value of 100 and $69 \%$ and a specificity of 100 and $84 \%$, respectively (Fig. 6B). Twenty PDXs were also tested with cisplatin. Responses were concordant with those to irinotecan in 18 of 20 cases (11 resistant and 7 responses), whereas two irinotecan responder PDXs did not respond to cisplatin (table S1).

To determine the prognostic relevance of SLNF11 expression in human TNBC, we analyzed SLNF11 mRNA expression by RT-PCR in 250 patients with TNBC (table S2). All patients received adjuvant $(n=242)$, neoadjuvant $(n=7)$ chemotherapy, or both $(n=1)$. Area under the curve (AUC) analysis was performed to identify a cut point to divide the TNBC cohort into low and high SLNF11 expression subgroups. Both metastasis-free survival (MFS) and overall survival (OS) of patients with low SLNF11-expressing tumors (70.5 and 76.1\%, respectively, at 5 years) were shorter than those of patients with high SLNF11-expressing tumors ( 82.3 and $87.9 \%$, respectively) (Fig. 6C). Multivariate analysis using a Cox proportional hazards model assessed the predictive value for MFS and OS of the parameters with a $P$ value $<0.1$ on univariate analysis, including lymph node status, 

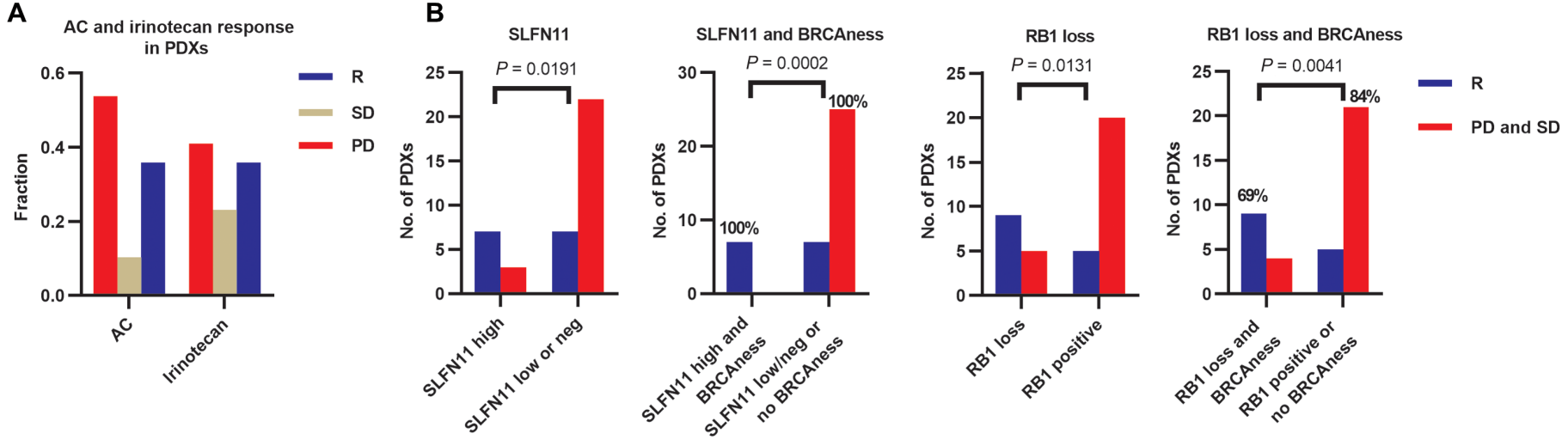

C
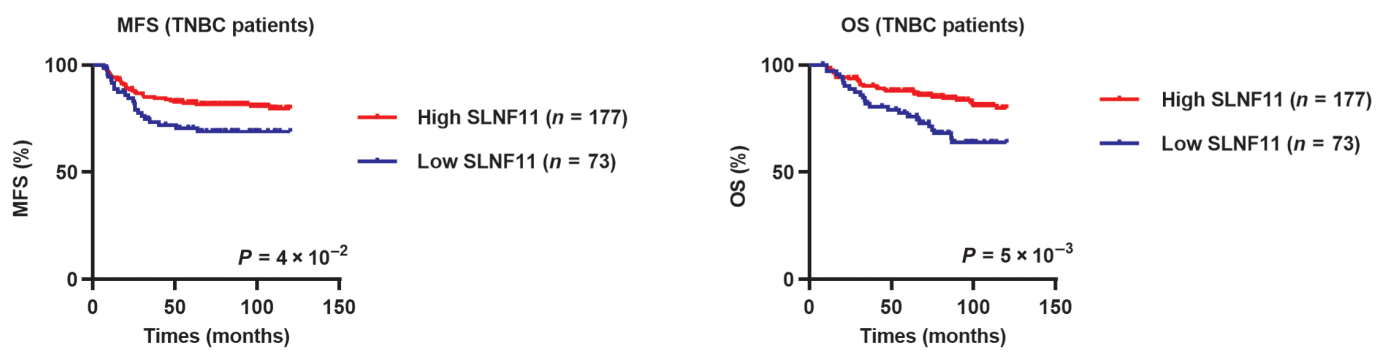

Fig. 6. SLFN1 1, RB1, and BRCAness and response to AC. (A) Response to AC and irinotecan in 39 TNBC PDXs (expressed as a fraction of the total tumor number). (B) Combination of SLFN1 1 expression, RB1 loss, and BRCAness as correlates of AC response in 39 TNBC PDXs. $P$ values were calculated with the Fisher's exact test. (C) MFS (metastasis-free survival). and OS of 250 patients with TNBC according to SLFN11 gene expression, determined by RT-PCR analysis. Survival distributions were estimated by the Kaplan-Meier method, and $P$ values were calculated with the log-rank test.

macroscopic tumor size, chemotherapy, hormonotherapy, molecular histology, and PIK3CA mutation status (table S3) and SLFN11 mRNA expression. The prognostic significance of lymph node status $(P<0.0001)$, molecular histology $(P=0.029)$, and $S L F N 11 \mathrm{mRNA}$ expression $(P=0.019)$ persisted for OS. This retrospective analysis confirms the value as SLFN11 expression as a potential marker of response to chemotherapy in patients with TNBC.

\section{DISCUSSION}

In this study, we found that $37 \%$ of TNBC PDXs responded to irinotecan with a partial or complete response and that an additional $22 \%$ of tumors showed SD. In the clinic, few studies with TOP1 inhibitors in BC have been published before 2017, and they included nonselected BC $(9,32)$. Recently, two clinical trials tested sacituzumab govitecan and sacituzumab govitecan-hziy in metastatic patients with TNBC with responses in 30 and $33 \%$ of patients, respectively $(33,34)$. Our findings are therefore similar to those obtained in those studies in terms of proportion of TNBC responding to irinotecan.

The second important finding of our study is the association of BRCA1/2 mutations and the genomic signature of BRCAness with irinotecan response. The HRD-LST score is highly correlated with defect in BRCA1/2 genes and is associated with impaired formation of RAD51 foci in treated tumors, a functional biomarker of HRmediated repair $(16,35)$. Analysis of $\gamma \mathrm{H} 2 \mathrm{AX}$ and RAD51 foci 24 hours after irinotecan treatment in two PDXs confirmed persistent DNA damage and lack of DNA repair by HR in the PDXs with BRCAness as compared to those without. In the clinical setting, the LST signature has one main advantage compared to the functional RAD51 assay:
It can be determined in baseline tumor samples and does not require posttreatment tumor biopsies or ex vivo irradiation of patients' tumor cells, protocols that have practical issues that limit their clinical applicability.

The association between defects in DNA repair by HR and high sensitivity to camptothecin was found in yeast and extended to mammalian models $(6,30,36-40)$. Similarly, the clinical indenoisoquinoline TOP1 inhibitors, LMP400 (indotecan) and LMP776 (indimitecan), were recently shown to exhibit selective activity in HRD (BRCAness) models alone and in combination with the PARP inhibitor olaparib (30).

Other potential markers associated with irinotecan response in our study are SLFN11 expression and RB1 loss. SLFN11 binds chromatin at stressed replication foci and irreversibly arrests replication (41). About $50 \%$ of human cancer cell lines inactivate SLFN11 by epigenetic regulation $(42,43)$ and are highly resistant to a broad range of widely used anticancer drugs including not only TOP1 inhibitors but also TOP2 inhibitors and cisplatin, gemcitabine, and hydroxyurea $(7,21)$. Yet, $50 \%$ of cancer cell lines that express SLFN11 are responsive to the above listed drugs. SFLN11 causes a replicative block in presence of DNA damage, resulting in tumor cell death, and works as an S-phase cell cycle checkpoint independent of ATR $(4,21)$. Consistently, in our study, PDXs with concomitant BRCAness phenotype and high expression of SLFN11 were high responders to irinotecan (complete response), whereas most tumors with BRCAness but low or no expression of SLFN11 showed intermediate response (SD) or resistance. Irinotecan was also highly efficient in a germline BRCA1-mutated and olaparib-resistant PDX, suggesting that TOP1 inhibitors could provide an alternative treatment strategy for patients with BRCA1-mutated tumors not responding to PARP inhibitors. 
Overall, our findings are in line with the notion that concomitant defects in DNA repair and checkpoints render cancer cells highly vulnerable to TOP1 inhibitors $(5,6,44)$. Accordingly, sensitivity to irinotecan was increased by an ATR inhibitor in SLFN11negative PDXs with BRCAness, further supporting a model where inhibition of S-phase checkpoint combined with a defect in HR results in increased sensitivity to irinotecan. Our study demonstrates that about $40 \%$ of TNBC PDXs present with high expression of SLFN11 and 22\% of TNBC PDXs present with high expression of SLFN11 combined with BRCAness, which is also the percentage found in TCGA TNBC (fig. S2).

In addition to SLFN11, RB1 loss was also associated with response to irinotecan. RB1 is a critical component of checkpoint signaling because it promotes $\mathrm{G}_{1}$-phase cell cycle arrest and limits replicative damage (24). The finding that the most irinotecan-sensitive PDXs are BRCAness positive, SLFN11 positive, and RB1 negative suggests a model where concomitant disruptions of both the $\mathrm{G}_{1}$ - and S-phase checkpoints not only drive tumor cell proliferation but also render cancer cells prone to lethal replicative damage when they are defective in HR (BRCAness) and express SLFN11 (fig. S3).

We show that the clinical indenoisoquinolines LMP400 (indotecan) and LMP776 (indimitecan) $(1,10,12,30)$ are highly efficient in irinotecan-responding models. In contrast to the camptothecin derivatives, the indenoisoquinolines are chemically stable, have long plasma half-life, and are not substrates for the adenosine 5 '-triphosphate-binding cassette drug efflux transporters $(1,12)$. Moreover, the indenoisoquinoline derivatives do not produce diarrhea in humans (12).

Last, there was a strong overlap between response to irinotecanand anthracycline-based chemotherapies and cisplatin in our PDXs, and the proposed markers were associated with $\mathrm{AC}$ response. Although TOP1 and TOP2 inhibitors differ in their primary targets (TOP1 versus TOP2), they both induce DNA DSBs (5). Because of the redundant pathways involved in the repair of DNA damage induced by TOP1 and TOP 2 inhibitors and platinum drugs, it is not unexpected to observe overlapping responses to these agents.

A limitation of our study is the lack of clinical samples from patients with $\mathrm{BC}$ treated with irinotecan or other TOP1 inhibitors. A retrospective analysis of tumor samples from patients with TNBC treated with sacituzumab govitecan $(33,34)$ could be an opportunity to validate the proposed markers in the clinical setting. Nevertheless, we could demonstrate that high SLFN11 expression was correlated with a better outcome in a cohort of patients with TNBC treated with anthracycline-based chemotherapy, and the results of a recent retrospective study in $\mathrm{BC}$ are consistent with our conclusions (22).

In conclusion, our study demonstrates that TNBCs with BRCAness phenotype, high SLFN11 expression, and concomitant defects in RB1 are highly sensitive to irinotecan and indenoisoquinoline TOP1 inhibitors. The proposed markers are easily analyzable in baseline tumor samples and could therefore be used to identify patients more likely to respond to TOP1 inhibitors in clinical trials testing agents such as antibody-drug conjugates of SN38, the active metabolite of irinotecan, or indenoisoquinoline derivatives. Validation of these proposed markers in a prospective clinical trial is warranted (1) because the activity of most cytotoxic agents cannot be predicted, resulting in a "trial and error" scenario with highly detrimental consequences in patients with rapidly growing tumors such as TNBC.

\section{MATERIALS AND METHODS Study design}

The objective of this study was to identify correlates of response to TOP1 inhibitors in PDXs of TNBC. Response to irinotecan was determined in vivo in 40 different PDX models. Potential markers were identified using Affymetrix gene expression arrays and validated by RT-PCR and IHC analyses. BRCA1/2 mutations were identified by targeted Next Generation Sequencing (NGS) analysis, and the BRCAness status was determined on the basis of the number of LST.

In in vivo experiments, mice were individually identified and randomly assigned to control or treated groups when tumors reached a volume of 60 to $200 \mathrm{~mm}^{3}$. The number of replicates included in each experiment is indicated in each figure legend. They were chosen to ensure adequate statistical power and were based on previous experience with the PDX models used in this study. The researchers were not blinded for any experiments.

\section{Patients and samples corresponding to TNBC PDXs}

The histopathological and clinical characteristics of the patients with TNBC corresponding to PDXs are summarized in Table 1. Tumor samples were obtained at surgery from primary BC (55\%), axillary lymph node metastases (5\%), or residual BC after neoadjuvant treatment (40\%). The mean age of patients was 56 (range: 29 to 89). According to the Tumor, Node, Metastasis (TNM) classification, 8 tumors (20\%) were T1, 19 tumors (48\%) were T2, 9 tumors (23\%) were T3, 2 tumors (5\%) were T4, and 2 were not determined. Most cancers $(58 \%)$ were N0 and M0 at surgery (98\%). Thirty-seven tumors were diagnosed as invasive carcinoma of no special type, and three tumors were metaplastic BC. Lymphovascular invasion (LVI) was present in $35 \%$ of patients, and most ( 39 of 40 ) were classified as grade 3 tumors according to the Scarff-Bloom-Richardson (SBR) grading. Axillary lymph nodes were positive in $41 \%$ of patients, and $42.5 \%$ of patients had distant recurrence after tumorectomy or mastectomy.

\section{Patients and samples of TNBC: Prognostic cohort $(n=250)$}

Samples from 250 patients with TNBC have been analyzed with informed consent. The study was approved by the local ethics committee (Breast Group of Institut Curie Hospital).

All patients (mean age 52.7 years, range: 27 to 81 years) met the following criteria: Primary unilateral nonmetastatic TNBC for which complete clinical, histological, and biological data were available; neoadjuvant and/or adjuvant chemotherapy and full follow-up at Institut Curie. Adjuvant therapy was administered to 243 patients and neoadjuvant chemotherapy to 8 patients. The histological type and the number of positive axillary nodes were established at the time of surgery. Standard prognostic factors are detailed in data file S2. During a median follow-up of 8 years, 56 patients developed distant metastasis. Ten specimens of adjacent normal breast tissue from patients with $\mathrm{BC}$ and normal breast tissue from women undergoing cosmetic breast surgery were used as sources of normal RNA.

\section{PDXs and in vivo preclinical assays}

PDXs were established from patients with TNBC with informed consent, in accordance with published protocols (13-15). End points for animal experiments were in accordance with Institutional Animal Care and French Committee-approved criteria (project authorization no. 02163.02). Female Swiss nude mice were purchased from Charles River and maintained under specific pathogen-free conditions. 
Irinotecan monotherapy was administered intraperitoneally weekly, at a dose of $50 \mathrm{mg} / \mathrm{kg}$, for up to 6 weeks or less if the tumors reached the ethical size limit $\left(1500 \mathrm{~mm}^{3}\right)$. In the combination study, irinotecan was given at $40 \mathrm{mg} / \mathrm{kg}$ at days 1,8 , and 15 and VE-822 (MedChemExpress) at $50 \mathrm{mg} / \mathrm{kg}$ at days $1,2,3,8,9,10,15,16$, and 17 . Olaparib was given at $100 \mathrm{mg} / \mathrm{kg}, 5$ days/week for 4 weeks. The indenoisoquinolines LMP400 and LMP776 were obtained from the National Cancer Institute (45) and were administered intraperitoneally 5 days/week at $10 \mathrm{mg} / \mathrm{kg}$ for 4 weeks (HBCX-60) or 3 weeks (HBCx-39). Doxorubicin (Adriamycin, Teva Pharmaceutical Industries Ltd.), cisplatin (CDDP, Teva Pharmaceutical Industries Ltd.), and cyclophosphamide (Endoxan, Baxter) were administered intraperitoneally at doses of 2,6 , and $100 \mathrm{mg} / \mathrm{kg}$, respectively, every 3 weeks.

When tumors reached a volume of 60 to $200 \mathrm{~mm}^{3}$, mice were individually identified and randomly assigned to the control or treated groups, and the treatments were started. Tumor growth was evaluated by measurement of two perpendicular diameters of tumors with a caliper twice per week. Individual tumor volumes were calculated as $V=a \times b^{2} / 2$, $a$ being the largest diameter and $b$ being the smallest. For each tumor, volumes were reported relative to the initial volume [as relative tumor volume (RTV)]. Means (and standard deviation) of RTVs in the same treatment group were calculated, and growth curves were established as a function of time. Optimal tumor growth inhibition of treated tumors versus controls was calculated as the ratio of the mean RTV in the treated group to the mean RTV in the control group at the same time. Mice were euthanized when the tumor volume reached the ethical limit of 1200 to $1500 \mathrm{~mm}^{3}$. Percent change in tumor volume was calculated for each tumor as [(Vf - V0)/ $\mathrm{V} 0]^{*} 100$, where $\mathrm{V} 0=$ initial volume (at the beginning of treatment) and $\mathrm{Vf}=$ final volume (at the end of treatment). Tumor regression (R) was defined as a decrease in tumor volume of at least $50 \%$, taking the baseline tumor volume as reference; at least a 35\% increase in tumor volume identified PD and responses that were between +35 and $-50 \%$ were considered as $\operatorname{SD}(13,14)$. Individual data (tumor volumes or relative tumor volumes) are in data file S4.

\section{SNP-based assay for BRCAness classification}

PDXs were profiled using Affymetrix genomics array: 18 PDXs with SNP 6.0 and 22 with CytoScan HD arrays, as previously described (15). Raw data were normalized with Genotyping Console (SNP 6.0 arrays) or Chromosome Analysis Suite (CytoScan HD arrays). CytoScan HD and SNP array (Affymetrix) data were processed using the Genome Alteration Print (GAP) methodology to obtain absolute copy number profiles (17). BRCAness classification was performed on the basis of the number of LSTs as previously detailed $(16,17)$.

\section{Microarray data analysis}

GeneChip Human 1.1 ST arrays were hybridized according to the Affymetrix recommendations, using the Ambion WT Expression Kit protocol (Life Technologies) and Affymetrix labeling and hybridization kits as detailed elsewhere. Affymetrix CEL files were imported into the Gene Expression Workflow in Partek Genomics Suite version 7.0 (Partek Inc., www.partek.com). Background correction, quantile normalization, $\log _{2}$ transformation, and probeset annotation were performed using default settings for the robust multichip average procedure. For the identification of differentially expressed genes, we used one-way analysis of variance (ANOVA), log fold changes in expression $>1.5$, and $P$ values $<0.05$ to be considered statistically significant.

\section{TCGA cohort}

FPKM (fragments per kilobase of exon model per million reads mapped) RNA sequencing data on SLFN11 expression were obtained from TCGA. FPKM were normalized to obtain $z$ scores. TNBCs were annotated using the set of genes associated with the estrogen receptor 1 (ESR1) pathway. Tumors with deleterious mutations in BRCA1/2 and RAD51 and loss of heterozygosity in corresponding loci, BRCA1, or RAD51 promoter methylation, and high genomic HRD score (LSTs) were considered to have BRCAness.

\section{Methylation of BRCA1}

We proceeded to sodium bisulfite modification of $100 \mathrm{ng}$ of genomic DNA, following the manufacturer's protocol (EpiTect Plus DNA Bisulfite Kit, QIAGEN). The methylated status of the BRCA1 promoter was determined by PCR with specific primers and verified by pyrosequencing (PyroMark Q96 ID Instrument, QIAGEN). The degree of $\mathrm{CpG}$ methylation was evaluated from the ratios of thymine and cytosine ( $\mathrm{T}$ and $\mathrm{C}$ ) in the sequence.

\section{RT-PCR analysis}

RNA extraction and RT quantitive PCR were performed as previously described (14). For gene normalization, we used the human TATA box-binding protein (TBP, GenBank accession no. NM_003194). Results are expressed as $\mathrm{n}$-fold differences in target gene expression relative to the TBP gene. In the prognostic cohort of 250 TNBCs, SLNF11 values of the tumor samples were subsequently normalized such that the median of the SLNF11 values for the 10 normal breast tissues was 1 .

\section{Western blotting}

Proteins were extracted as described previously (8). Lysates were resolved on 4 to $12 \%$ TGX gels (Bio-Rad), transferred into nitrocellulose membranes (Bio-Rad), and immunoblotted with rabbit antibodies against $\gamma \mathrm{H} 2 \mathrm{AX}, \mathrm{P}-\mathrm{CHK} 1\left(\mathrm{Ser}^{345}\right)$, P-CHK2 $\left(\mathrm{Thr}^{68}\right)$, and Ku80, all purchased from Cell Signaling Technology. After washes, membranes were incubated with horseradish peroxidase-conjugated affinitypurified goat anti-rabbit secondary antibodies (111-035-045, Jackson ImmunoResearch Laboratories Inc., Interchim).

\section{Immunohistochemistry}

Xenografted tumors were fixed in $10 \%$ neutral buffered formalin, embedded in paraffin, and stained with hematoxylin and eosin. Immunostaining was performed on a DISCOVERY XT Platform (Ventana Medical Systems, part of Roche Diagnostics). The slides were incubated with a monoclonal mouse antibody against RB1 (no. 9309, clone 4H1, Cell Signaling Technology) and a polyclonal rabbit antibody against SLFN11 (no. HPA023030, Sigma-Adrich). Slides immunostained with mouse and rabbit immunoglobulin $\mathrm{G}$ (IgG) were used as negative controls. Slides were incubated with anti-rabbit/mouse secondary antibodies (horseradish peroxidase complex) and $\mathrm{DAB}$ (3,3'-diaminobenzidine tetrahydrochloride) as the substrate for color development (ChromoMap Kit with anti-rabbit OmniMap, Ventana Medical Systems). Immunostaining of RB1 was performed as detailed in previous works $(14,28)$. Expression of SLFN11 was quantified with the $H$-score: Sections were scored for intensity ( 0 to $3+$ ) and extent ( 0 to $100 \%$ ) of staining. By multiplying intensity and extent of staining, each tumor was assigned an $\mathrm{H}$-score (range: 0 to 300). We considered a tumor SLFN11 negative with $H$-score $=0$, SLFN11 low with an $H$-score between 1 and 60, and SLFN11 high when the $H$-score was higher than 60 . 


\section{RAD51 immunofluorescence assay}

RAD51 scores were assessed by immunofluorescence analysis in untreated and treated xenografts ( 24 hours after a single treatment) following the protocol published by Graeser et al. (19). Immunofluorescence was carried out on 5-mm sections of formalin-fixed and paraffin-embedded tumor tissues. After antigen retrieval by microwaving at $\mathrm{pH} 9$ [10 $\mathrm{mM}$ tris/1 mM EDTA (pH 9) buffer] for $18 \mathrm{~min}$ followed by $20 \mathrm{~min}$ cooling in buffer, sections were treated with Triton X-100 $0.2 \%$ for permeabilization for $20 \mathrm{~min}$, washed in phosphate-buffered saline (PBS)-Tween $200.1 \%$, and blocked with immunofluorescence buffer (IFF, 3\% bovine serum albumin and $5 \%$ donkey serum in PBS-Tween 20 0.1\%) for $30 \mathrm{~min}$ at room temperature. Sections were stained with a rabbit anti-geminin antibody (ProteinTech no. 10802-1-AP) and a mouse anti-RAD51 antibody (GeneTex no. GTX70230) in IFF overnight at $4^{\circ} \mathrm{C}$, washed with PBS-Tween 20 0.1\%, and stained with Alexa Fluor 594 donkey anti-rabbit IgG (Life Technologies, Thermo Fisher Scientific no. A-21207) and Alexa Fluor 488 donkey anti-mouse IgG (Life Technologies, Thermo Fisher Scientific no. A-21202) for 1 hour at room temperature. Last, slides were washed in PBS-Tween $200.1 \%$ and mounted with Fluoroshield with 4',6-diamidino-2-phenylindole histology mounting medium (Sigma-Aldrich). Images were captured with a Leica DM6000 B microscope. Between 100 and 200, tumor cells were counted in 10 representative areas across the section. The RAD51 score was assessed as the percentage of geminin-positive cells that were also positive for RAD51. A cell was considered RAD51 positive if it had at least 10 nuclear foci.

\section{Statistical analyses PDX studies}

Categorical variables were analyzed with the Fisher's exact test. Sensitivity, specificity, and positive and negative predictive values were calculated with GraphPad Prism software. Two-tailed unpaired $t$ tests were used when comparing two groups. Pearson's correlation coefficient was used to assess correlation between SLFN11 gene and protein expression. The frequency distribution of SLFN11 $\mathrm{H}$-score across PDX models was calculated with GraphPad Prism software.

\section{Patient cohort}

To visualize the efficacy of SLNF11 mRNA expression for discriminating two populations (patients who developed/did not develop metastases) in the absence of an arbitrary cut-off value, data were summarized in a receiver operating characteristic curve. The AUC was calculated as a single measure to discriminate efficacy. MFS was determined as the interval between initial diagnosis and detection of the first metastasis. OS was determined as the interval between initial diagnosis and the date of death. Survival distributions were estimated by the Kaplan-Meier method, and the significance of differences between survival rates was ascertained with the log-rank test. The Cox proportional hazards regression model was used to assess prognostic significance, and the results are presented as hazard ratios and $95 \%$ confidence intervals.

\section{SUPPLEMENTARY MATERIALS}

stm.sciencemag.org/cgi/content/full/12/531/eaax2625/DC1

Fig. S1. Transcriptomic expression of TOP1 and MDR1 genes in PDX models.

Fig. S2. Expression of SLFN11 in TGCA TNBC.

Fig. S3. HR-mediated repair and DNA damage checkpoint activation in response to TOPI and TOPII inhibitors.

Table S1. Comparison of cisplatin and irinotecan responses in 20 PDXs.

Table S2. Characteristics of the 250 TNBCs.
Table S3. Multivariate COX analysis of OS for SLFN11 mRNA expression in the series of 250 TNBCs.

Data file S1. Clinical and pathological characteristics of the TNBCs corresponding to PDXs. Data file S2. Characteristics of TNBC PDXs (BRCAness, BRCA1/2 mutations, RB1, and SLFN1 1 status).

Data file S3. List of genes differentially expressed between PDXs in the "response group (R)" as compared to "progressive disease (PD)" group.

Data file S4. Individual data points.

View/request a protocol for this paper from Bio-protocol.

\section{REFERENCES AND NOTES}

1. A. Thomas, Y. Pommier, Targeting topoisomerase i in the era of precision medicine. Clin. Cancer Res. 25, 6581-6589 (2019).

2. Y. Pommier, Topoisomerase I inhibitors: Camptothecins and beyond. Nat. Rev. Cancer $\mathbf{6}$, 789-802 (2006).

3. Y. Pommier, M. J. O'Connor, J. de Bono, Laying a trap to kill cancer cells: PARP inhibitors and their mechanisms of action. Sci. Transl. Med. 8, 362ps317 (2016).

4. J. Murai, S. W. Tang, E. Leo, S. A. Baechler, C. E. Redon, H. Zhang, M. Al Abo, V. N. Rajapakse, E. Nakamura, L. M. M. Jenkins, M. I. Aladjem, Y. Pommier, SLFN11 blocks stressed replication forks independently of ATR. Mol Cell 69, 371-384 (2018).

5. Y. Pommier, Y. Sun, S.-N. Huang, J. L. Nitiss, Roles of eukaryotic topoisomerases in transcription, replication and genomic stability. Nat. Rev. Mol. Cell Biol. 17, 703-721 (2016).

6. Y. Pommier, J. M. Barcelo, V. A. Rao, O. Sordet, A. G. Jobson, L. Thibaut, Z. H. Miao, J. A. Seiler, H. Zhang, C. Marchand, K. Agama, J. L. Nitiss, C. Redon, Repair of topoisomerase I-mediated DNA damage. Prog. Nucleic Acid Res. Mol. Biol. 81, 179-229 (2006).

7. G. Zoppoli, M. Regairaz, E. Leo, W. C. Reinhold, S. Varma, A. Ballestrero, J. H. Doroshow, Y. Pommier, Putative DNA/RNA helicase Schlafen-11 (SLFN11) sensitizes cancer cells to DNA-damaging agents. Proc. Natl. Acad. Sci. U.S.A. 109, 15030-15035 (2012).

8. M. H. Kang, J. Wang, M. R. Makena, J.-S. Lee, N. Paz, C. P. Hall, M. M. Song, R. I. Calderon, R. E. Cruz, A. Hindle, W. Ko, J. B. Fitzgerald, D. C. Drummond, T. J. Triche, C. P. Reynolds, Activity of MM-398, nanoliposomal irinotecan (nal-IRI), in Ewing's family tumor xenografts is associated with high exposure of tumor to drug and high SLFN11 expression. Clin. Cancer Res. 21, 1139-1150 (2015).

9. I. Kümler, N. Brünner, J. Stenvang, E. Balslev, D. L. Nielsen, A systematic review on topoisomerase 1 inhibition in the treatment of metastatic breast cancer. Breast Cancer Res. Treat. 138, 347-358 (2013).

10. J. H. Burton, C. Mazcko, A. LeBlanc, J. M. Covey, J. Ji, R. J. Kinders, R. E. Parchment, C. Khanna, M. Paoloni, S. Lana, K. Weishaar, C. London, W. Kisseberth, E. Krick, D. Vail, M. Childress, J. N. Bryan, L. Barber, E. J. Ehrhart, M. Kent, T. Fan, K. Kow, N. Northup, H. Wilson-Robles, J. Tomaszewski, J. L. Holleran, M. Muzzio, J. Eiseman, J. H. Beumer, J. H. Doroshow, Y. Pommier, $\mathrm{NCl}$ comparative oncology program testing of noncamptothecin indenoisoquinoline topoisomerase I inhibitors in naturally occurring canine lymphoma. Clin. Cancer Res. 24, 5830-5840 (2018).

11. Y. Pommier, E. Leo, H. L. Zhang, C. Marchand, DNA topoisomerases and their poisoning by anticancer and antibacterial drugs. Chem. Biol. 17, 421-433 (2010).

12. S. Kummar, A. Chen, M. Gutierrez, T. D. Pfister, L. Wang, C. Redon, W. M. Bonner, W. Yutzy, Y. Zhang, R. J. Kinders, J. Ji, D. Allen, J. M. Covey, J. L. Eiseman, J. L. Holleran, J. H. Beumer, L. Rubinstein, J. Collins, J. Tomaszewski, R. Parchment, Y. Pommier, J. H. Doroshow, Clinical and pharmacologic evaluation of two dosing schedules of indotecan (LMP400), a novel indenoisoquinoline, in patients with advanced solid tumors. Cancer Chemother. Pharmacol. 78, 73-81 (2016).

13. E. Marangoni, A. Vincent-Salomon, N. Auger, A. Degeorges, F. Assayag, P. de Cremoux, L. de Plater, C. Guyader, G. De Pinieux, J.-G. Judde, M. Rebucci, C. Tran-Perennou, X. Sastre-Garau, B. Sigal-Zafrani, O. Delattre, V. Diéras, M. F. Poupon, A new model of patient tumor-derived breast cancer xenografts for preclinical assays. Clin. Cancer Res. 13, 3989-3998 (2007)

14. E. Marangoni, C. Laurent, F. Coussy, R. El-Botty, S. Château-Joubert, J.-L. Servely, L. de Plater, F. Assayag, A. Dahmani, E. Montaudon, F. Nemati, J. Fleury, S. Vacher, D. Gentien, A. Rapinat, P. Foidart, N. E. Sounni, A. Noel, A. Vincent-Salomon, M. Lae, D. Decaudin, S. Roman-Roman, I. Bièche, M. Piccart, F. Reyal, Capecitabine efficacy is correlated with TYMP and RB1 expression in PDX established from triple-negative breast cancers. Clin. Cancer Res. 24, 2605-2615 (2018).

15. F. Coussy, L. de Koning, M. Lavigne, V. Bernard, B. Ouine, A. Boulai, R. El Botty, A. Dahmani, E. Montaudon, F. Assayag, L. Morisset, L. Huguet, L. Sourd, P. Painsec, C. Callens, S. Chateau-Joubert, J.-L. Servely, T. Larcher, C. Reyes, E. Girard, G. Pierron, C. Laurent, S. Vacher, S. Baulande, S. Melaabi, A. Vincent Salomon, D. Gentien, V. Dieras, I. Bieche, E. Marangoni, A large collection of integrated genomically characterized patient-derived xenografts highlighting the heterogeneity of triple-negative breast cancer. Int. J. Cancer 145, 1902-1912 (2019).

16. T. Popova, E. Manié, G. Rieunier, V. Caux-Moncoutier, C. Tirapo, T. Dubois, O. Delattre, B. Sigal-Zafrani, M. Bollet, M. Longy, C. Houdayer, X. Sastre-Garau, A. Vincent-Salomon, D. Stoppa-Lyonnet, M.-H. Stern, Ploidy and large-scale genomic instability consistently 
identify basal-like breast carcinomas with BRCA1/2 inactivation. Cancer Res. 72, 5454-5462 (2012).

17. T. Popova, E. Manié, D. Stoppa-Lyonnet, G. Rigaill, E. Barillot, M. H. Stern, Genome alteration Print (GAP): A tool to visualize and mine complex cancer genomic profiles obtained by SNP arrays. Genome Biol. 10, R128 (2009).

18. M. M. Hoppe, R. Sundar, D. S. P. Tan, A. D. Jeyasekharan, Biomarkers for homologous recombination deficiency in cancer. J. Natl. Cancer Inst. 110, 704-713 (2018).

19. M. Graeser, A. McCarthy, C. J. Lord, K. Savage, M. Hills, J. Salter, N. Orr, M. Parton, I. E. Smith, J. S. Reis-Filho, M. Dowsett, A. Ashworth, N. C. Turner, A marker of homologous recombination predicts pathologic complete response to neoadjuvant chemotherapy in primary breast cancer. Clin. Cancer Res. 16, 6159-6168 (2010).

20. J. Barretina, G. Caponigro, N. Stransky, K. Venkatesan, A. A. Margolin, S. Kim, C. J. Wilson, J. Lehár, G. V. Kryukov, D. Sonkin, A. Reddy, M. Liu, L. Murray, M. F. Berger, J. E. Monahan, P. Morais, J. Meltzer, A. Korejwa, J. Jané-Valbuena, F. A. Mapa, J. Thibault, E. Bric-Furlong, P. Raman, A. Shipway, I. H. Engels, J. Cheng, G. K. Yu, J. Yu, P. Aspesi Jr., M. de Silva, K. Jagtap, M. D. Jones, L. Wang, C. Hatton, E. Palescandolo, S. Gupta, S. Mahan, C. Sougnez, R. C. Onofrio, T. Liefeld, L. MacConaill, W. Winckler, M. Reich, N. Li, J. P. Mesirov, S. B. Gabriel, G. Getz, K. Ardlie, V. Chan, V. E. Myer, B. L. Weber, J. Porter, M. Warmuth, P. Finan, J. L. Harris, M. Meyerson, T. R. Golub, M. P. Morrissey, W. R. Sellers, R. Schlegel, L. A. Garraway, The cancer cell line encyclopedia enables predictive modelling of anticancer drug sensitivity. Nature 483, 603-607 (2012).

21. J. Murai, A. Thomas, M. Miettinen, Y. Pommier, Schlafen 11 (SLFN11), a restriction factor for replicative stress induced by DNA-targeting anti-cancer therapies. Pharmacol. Ther. 201, 94-102 (2019).

22. E. Isnaldi, D. Ferraioli, L. Ferrando, S. Brohée, F. Ferrando, P. Fregatti, D. Bedognetti, A. Ballestrero, G. Zoppoli, Schlafen-11 expression is associated with immune signatures and basal-like phenotype in breast cancer. Breast Cancer Res. Treat. 177, 335-343 (2019).

23. A. Ertel, J. L. Dean, H. Rui, C. Liu, A. K. Witkiewicz, K. E. Knudsen, E. S. Knudsen, RB-pathway disruption in breast cancer: differential association with disease subtypes, diseasespecific prognosis and therapeutic response. Cell Cycle 9, 4153-4163 (2010).

24. K. E. Knudsen, D. Booth, S. Naderi, Z. Sever-Chroneos, A. F. Fribourg, I. C. Hunton, J. R. Feramisco, J. Y. Wang, E. S. Knudsen, RB-dependent S-phase response to DNA damage. Mol. Cell. Biol. 20, 7751-7763 (2000).

25. K. R. Stengel, J. L. Dean, S. L. Seeley, C. N. Mayhew, E. S. Knudsen, RB status governs differential sensitivity to cytotoxic and molecularly-targeted therapeutic agents. Cell Cycle 7, 1095-1103 (2008).

26. E. E. Bosco, Y. Wang, H. Xu, J. T. Zilfou, K. E. Knudsen, B. J. Aronow, S. W. Lowe, E. S. Knudsen, The retinoblastoma tumor suppressor modifies the therapeutic response of breast cancer. J. Clin. Invest. 117, 218-228 (2007).

27. A. K. Witkiewicz, E. S. Knudsen, Retinoblastoma tumor suppressor pathway in breast cancer: prognosis, precision medicine, and therapeutic interventions. Breast Cancer Res. 16, 207 (2014).

28. P. Foidart, C. Yip, J. Radermacher, S. Blacher, M. Lienard, L. Montero-Ruiz, E. Maquoi, E. Montaudon, S. Chateau-Joubert, J. Collignon, M. Coibion, V. Jossa, E. Marangoni, A. Noël, N. E. Sounni, G. Jerusalem, Expression of MT4-MMP, EGFR, and RB in triple-negative breast cancer strongly sensitizes tumors to erlotinib and palbociclib combination therapy. Clin. Cancer Res. 25, 1838-1850 (2019).

29. J. Smith, L. M. Tho, N. Xu, D. A. Gillespie, The ATM-Chk2 and ATR-Chk1 pathways in DNA damage signaling and cancer. Adv. Cancer Res. 108, 73-112 (2010).

30. L. Marzi, L. Szabova, M. Gordon, Z. Weaver Ohler, S. K. Sharan, M. L. Beshiri, M. Etemadi, J. Murai, K. Kelly, Y. Pommier, The indenoisoquinoline TOP1 inhibitors selectively target homologous recombination-deficient and schlafen 11-positive cancer cells and synergize with olaparib. Clin. Cancer Res. 25, 6206-6216 (2019).

31. Early Breast Cancer Trialists' Collaborative Group (EBCTCG), R. Peto, C. Davies, J. Godwin, R. Gray, H. C. Pan, M. Clarke, D. Cutter, S. Darby, P. McGale, C. Taylor, Y. C. Wang, J. Bergh, A. Di Leo, K. Albain, S. Swain, M. Piccart, K. Pritchard, Comparisons between different polychemotherapy regimens for early breast cancer: Meta-analyses of long-term outcome among 100,000 women in 123 randomised trials. Lancet 379, 432-444 (2012).

32. E. A. Perez, D. W. Hillman, J. A. Mailliard, J. N. Ingle, J. M. Ryan, T. R. Fitch, K. M. Rowland, C. G. Kardinal, J. E. Krook, J. W. Kugler, S. R. Dakhil, Randomized phase II study of two irinotecan schedules for patients with metastatic breast cancer refractory to an anthracycline, a taxane, or both. J. Clin. Oncol. 22, 2849-2855 (2004).

33. A. Bardia, I. A. Mayer, J. R. Diamond, R. L. Moroose, S. J. Isakoff, A. N. Starodub, N. C. Shah, J. O'Shaughnessy, K. Kalinsky, M. Guarino, V. Abramson, D. Juric, S. M. Tolaney, J. Berlin, W. A. Messersmith, A. J. Ocean, W. A. Wegener, P. Maliakal, R. M. Sharkey, S. V. Govindan, D. M. Goldenberg, L. T. Vahdat, Efficacy and safety of anti-Trop-2 antibody drug conjugate sacituzumab govitecan (IMMU-132) in heavily pretreated patients with metastatic triple-negative breast cancer. J. Clin. Oncol. 35, 2141-2148 (2017).

34. A. Bardia, I. A. Mayer, L. T. Vahdat, S. M. Tolaney, S. J. Isakoff, J. R. Diamond, J. O'Shaughnessy, R. L. Moroose, A. D. Santin, V. G. Abramson, N. C. Shah, H. S. Rugo, D. M. Goldenberg, A. M. Sweidan, R. lannone, S. Washkowitz, R. M. Sharkey, W. A. Wegener, K. Kalinsky, Sacituzumab govitecan-hziy in refractory metastatic triple-negative breast cancer. N. Engl. J. Med. 380, 741-751 (2019).
35. R. W. Mutter, N. Riaz, C. K. Ng, R. Delsite, S. Piscuoglio, M. Edelweiss, L. G. Martelotto, R. A. Sakr, T. A. King, D. D. Giri, M. Drobnjak, E. Brogi, R. Bindra, G. Bernheim, R. S. Lim, P. Blecua, A. Desrichard, D. Higginson, R. Towers, R. Jiang, W. Lee, B. Weigelt, J. S. Reis-Filho, S. N. Powell, Bi-allelic alterations in DNA repair genes underpin homologous recombination DNA repair defects in breast cancer. J. Pathol. 242, 165-177 (2017).

36. W. K. Eng, L. Faucette, R. K. Johnson, R. Sternglanz, Evidence that DNA topoisomerase I is necessary for the cytotoxic effects of camptothecin. Mol. Pharmacol. 34, 755-760 (1988).

37. J. Nitiss, J. C. Wang, DNA topoisomerase-targeting antitumor drugs can be studied in yeast. Proc. Natl. Acad. Sci. U.S.A. 85, 7501-7505 (1988).

38. C. Liu, J. J. Pouliot, H. A. Nash, Repair of topoisomerase I covalent complexes in the absence of the tyrosyl-DNA phosphodiesterase Tdp1. Proc. Natl. Acad. Sci. U.S.A. 99, 14970-14975 (2002).

39. A. Fedier, R. A. Steiner, V. A. Schwarz, L. Lenherr, U. Haller, D. Fink, The effect of loss of Brca1 on the sensitivity to anticancer agents in p53-deficient cells. Int. J. Oncol. 22, 1169-1173 (2003).

40. J. L. Boerner, N. Nechiporchik, K. L. Mueller, L. Polin, L. Heilbrun, S. A. Boerner, G. L. Zoratti, K. Stark, P. M. LoRusso, A. Burger, Protein expression of DNA damage repair proteins dictates response to topoisomerase and PARP inhibitors in triple-negative breast cancer. PLOS One 10, e0119614 (2015).

41. Y. Mu, J. Lou, M. Srivastava, B. Zhao, X. H. Feng, T. Liu, J. Chen, J. Huang, SLFN11 inhibits checkpoint maintenance and homologous recombination repair. EMBO Rep. 17, 94-109 (2016).

42. S. W. Tang, A. Thomas, J. Murai, J. B. Trepel, S. E. Bates, V. N. Rajapakse, Y. Pommier, Overcoming resistance to DNA-targeted agents by epigenetic activation of schlafen 11 (SLFN11) expression with class I histone deacetylase inhibitors. Clin. Cancer Res. 24, 1944-1953 (2018).

43. E. E. Gardner, B. H. Lok, V. E. Schneeberger, P. Desmeules, L. A. Miles, P. K. Arnold, A. Ni, I. Khodos, E. de Stanchina, T. Nguyen, J. Sage, J. E. Campbell, S. Ribich, N. Rekhtman, A. Dowlati, P. P. Massion, C. M. Rudin, J. T. Poirier, Chemosensitive relapse in small cell lung cancer proceeds through an EZH2-SLFN11 axis. Cancer Cell 31, 286-299 (2017).

44. Y. Pommier, J. N. Weinstein, M. I. Aladjem, K. W. Kohn, Chk2 molecular interaction map and rationale for Chk2 inhibitors. Clin. Cancer Res. 12, 2657-2661 (2006).

45. L. Marzi, K. Agama, J. Murai, S. Difilippantonio, A. James, C. J. Peer, W. D. Figg, D. Beck, M. S. A. Elsayed, M. Cushman, Y. Pommier, Novel fluoroindenoisoquinoline noncamptothecin topoisomerase I inhibitors. Mol. Cancer Ther. 17, 1694-1704 (2018).

Acknowledgments: We thank M. Piccart for critical reading of the manuscript. Funding: Y.P. acknowledges the support of the Center for Cancer Research, the Intramural Program of the National Cancer Institute (BC-01-006161). E. Marangoni and Y.P. were supported by grants from PIC3i NCI-Curie and Site de Recherche Intégrée sur le Cancer (SIRIC2) (INCa-DGOS-Inserm_12554). Author contributions: F.C. performed the molecular analysis of PDXs, the clinical analysis, and annotation of patient clinical records. E. Marangoni conceived and supervised the experiments and wrote the paper. R.E.-B. performed Western blot experiments. S.C.-J., J.-L.S., P.F., N.E.S., L.F., and A.N. performed IHC analyses. A.D., E. Montaudon, L.M., P.P., L.S., L.H., and F.N. established the PDXs and performed in vivo experiments. S.L. performed immunofluorescence analysis. T.L. and A.S. performed the histological analysis of PDXs. C.R., P.L.R., and G.L. performed the genomic profiling of PDXs including the determination of BRCAness. S.V. and A.B. performed the RT-PCR analysis. T.P. and M.-H.S. conceived the LST signature of BRCAness used in this study. D.D., F.R., C.M., P.T.B., J.J., and I.B. contributed with a critical review of the manuscript. M.-F.P. contributed to the study design, and Y.P. helped in the study conception and manuscript editing. Competing interests: Y.P. is an inventor on NIH patents for LMP400 and LMP776: US 8,053,443 B2; US 8,829,022 B2; US 9,217,010 B2; US 9,388,211 B2; US 9,399,660 B2; JP541211382; JP5567157 B2; EP1960366 B1, WO2007059008 A2. The title of the patents is "N-substituted indenoisoquinolines and syntheses thereof." T.P. and M.-H.S. are coinventors of the LST method for detecting inactivation of the HR pathway (BRCA1/2) in human tumors, patent numbers 20170260588 and 20150140122, under exclusive licensing with Myriad Genetics. Data and materials availability: Copy number Affymetrix data have been deposited in the ArrayExpress database at EMBL-EBI (www.ebi.ac.uk/arrayexpress) under accession numbers MTAB-8653 (CytoScan HD arrays) and E-MTAB-8654 (SNP6 arrays). Gene expression Affymetrix data have been deposited in Gene Expression Omnibus database under accession number GSE142639. All other data associated with this study are present in the paper or the Supplementary Materials.

Submitted 12 March 2019 Resubmitted 17 October 2019 Accepted 16 January 2020 Published 19 February 2020 $10.1126 /$ scitranslmed.aax2625

Citation: F. Coussy, R. El-Botty, S. Château-Joubert, A. Dahmani, E. Montaudon, S. Leboucher, L. Morisset, P. Painsec, L. Sourd, L. Huguet, F. Nemati, J.-L. Servely, T. Larcher, S. Vacher, A. Briaux, C. Reyes, P. La Rosa, G. Lucotte, T. Popova, P. Foidart, N. E. Sounni, A. Noel, D. Decaudin, L. Fuhrmann, A. Salomon, F. Reyal, C. Mueller, P. Ter Brugge, J. Jonkers, M.-F. Poupon, M.-H. Stern, I. Bièche, Y. Pommier, E. Marangoni, BRCAness, SLFN11, and RB1 loss predict response to topoisomerase I inhibitors in triple-negative breast cancers. Sci. Transl. Med. 12, eaax2625 (2020). 


\section{Science Translational Medicine}

\section{BRCAness, SLFN11, and RB1 loss predict response to topoisomerase I inhibitors in triple-negative breast cancers}

Florence Coussy, Rania El-Botty, Sophie Château-Joubert, Ahmed Dahmani, Elodie Montaudon, Sophie Leboucher, Ludivine Morisset, Pierre Painsec, Laura Sourd, Léa Huguet, Fariba Nemati, Jean-Luc Servely, Thibaut Larcher, Sophie Vacher, Adrien Briaux, Cécile Reyes, Philippe La Rosa, Georges Lucotte, Tatiana Popova, Pierre Foidart, Nor Eddine Sounni, Agnès Noel, Didier Decaudin, Laetitia Fuhrmann, Anne Salomon, Fabien Reyal, Christopher Mueller, Petra Ter Brugge, Jos Jonkers, Marie-France Poupon, Marc-Henri Stern, Ivan Bièche, Yves Pommier and Elisabetta Marangoni

Sci Transl Med 12, eaax2625.

DOI: 10.1126/scitranslmed.aax2625

\section{Starting at the TOP for cancer treatment}

Topoisomerase (TOP) inhibitors are chemotherapeutic drugs that cause DNA double-strand breaks during DNA replication. These can be repaired by homologous recombination, but some tumors, such as triple-negative breast cancer, have defects in proteins needed for this type of repair. By examining 40 patient-derived xenograft models of triple-negative breast cancer, Coussy et al. identified three key features associated with response to topoisomerase inhibitors in these tumors. The authors characterized the extent of DNA damage in cancer cells in the presence or absence of their proposed markers and tested a potential intervention for increasing drug sensitivity in tumors missing one of the three target features.

ARTICLE TOOLS

SUPPLEMENTARY MATERIALS

RELATED

REFERENCES

PERMISSIONS http://stm.sciencemag.org/content/12/531/eaax2625

http://stm.sciencemag.org/content/suppl/2020/02/14/12.531.eaax2625.DC1 http://stm.sciencemag.org/content/scitransmed/11/488/eaav0936.full http://stm.sciencemag.org/content/scitransmed/11/513/eaax9364.full http://stm.sciencemag.org/content/scitransmed/11/504/eaax7392.full http://stm.sciencemag.org/content/scitransmed/10/422/eaao1641.full

This article cites 45 articles, 19 of which you can access for free http://stm.sciencemag.org/content/12/531/eaax2625\#BIBL

http://www.sciencemag.org/help/reprints-and-permissions

Science Translational Medicine (ISSN 1946-6242) is published by the American Association for the Advancement of Science, 1200 New York Avenue NW, Washington, DC 20005. The title Science Translational Medicine is a registered trademark of AAAS.

Copyright @ 2020 The Authors, some rights reserved; exclusive licensee American Association for the Advancement of Science. No claim to original U.S. Government Works 